\title{
Phase Diagram of Nematic Polymer Monolayers with the Onsager Interaction Potential
}

\author{
Hongyun Wang ${ }^{1, *}$ and Hong Zhou ${ }^{2}$ \\ ${ }^{1}$ Department of Applied Mathematics and Statistics, University of California, Santa Cruz, CA 95064, USA \\ ${ }^{2}$ Department of Applied Mathematics, Naval Postgraduate School, Monterey, CA 93943, USA
}

\begin{abstract}
We present an asymptotic study on the phase diagram of two-dimensional nematic liquid crystal polymer monolayers with the Onsager intermolcular potential. In contrast to the case of MaierSaupe interaction potential where there is only one nematic branch, our analysis reveals that there are infinite many nematic branches in the case of the Onsager interaction potential. An asymptotic expression is derived for each nematic branch. For small polymer concentration the isotropic branch is the only equilibrium state. As the polymer concentration is increased, nematic branches appear one by one, starting with the first nematic branch. The polymer orientation distribution of the first nematic branch has a two fold rotational symmetry, the second branch has a four fold rotational symmetry, the third branch has a six fold rotational symmetry, and so on. To determine the stability of these nematic branches, we derive an asymptotic expression of free energy for each nematic branch. We find that free energies of all nematic branches are lower than that of the isotropic state, and the first nematic branch has the lowest free energy among all branches. To further investigate the stability and meta-stability, we carry out asymptotic analysis of the free energy when each nematic state is perturbed. We conclude that (1) the isotropic branch is stable until the first nematic branch appears, (2) the first nematic branch is stable, and (3) the isotropic branch (after the appearance of the first nematic branch) and all other nematic branches are unstable when perturbed by the leading Fourier mode in the first nematic branch. We also present a spectrum numerical method for calculating nematic branches and free energies. The spectrum method yields results that are accurate up to the computer precision. All of asymptotic results are confirmed by numerical results obtained with the spectrum method.
\end{abstract}

Keywords: Nematic Polymers, Phase Diagram, Isotropic-Nematic Phase Transition, Onsager Intermolecular Potential, Maier-Saupe Intermolecular Potential, Smoluchowski Equation, Free Energy of a Polymer Ensemble, Stability of an Equilibrium State.

\section{INTRODUCTION}

Microstructures play a significant role in understanding the phase transition for dispersions of rodlike macromolecules in liquid crystal polymers with or without external field. In 1949 Onsager developed the first theory that demonstrated an isotropic-nematic (IN) phase transition due to steric interactions alone. ${ }^{33}$ His theory was based on a virial expansion which ended up with a complicated nonlinear integral equation for the orientation distribution function. By using a nematic potential ("Onsager potential") and choosing an appropriate trial function for the orientation distribution function, Onsager was able to argue that when the polymer concentration is high enough, there is a transition from a uniform isotropic

\footnotetext{
*Author to whom correspondence should be addressed.
}

state to an orientationally ordered prolate nematic state. The Onsager theory is valid for dilute polymer solutions because the virial expansion is terminated at the second virial coefficients.

Many theories have been formulated after Onsager's work. For an introduction to the theoretical advances of liquid crystalline polymers we refer the reader to the review paper by Rey and Denn. ${ }^{34}$ Among many theories the DoiHess kinetic model ${ }^{6,20}$ is gaining popularity in recent years for describing various properties of liquid crystalline polymers (LCPs) in a solvent. This well-known kinetic theory was first developed by Doi and Edwards to describe spatially homogeneous flows of rodlike LCPs. The significance of the Doi-Hess theory lies on the fact that it models the ensemble of rodlike macromolecules using a meso-scale probability density function. Thus, it can 
accommodate the isotropic state, the nematic state, and the phase transition between them. In the Doi-Hess kinetic model, the intermolecular interaction is usually modeled by the Maier-Saupe potential because of the simplicity of the Maier-Saupe potential. The Maier-Saupe potential is an approximation to the Onsager potential. In the Maier-Saupe potential, the effect of the polymer ensemble on one polymer rod is represented by the second moment of the probability density. Thus, with the Maier-Saupe potential, an equilibrium state is completely determined by the second moment, which motivates the idea of following the evolution of only the second moment with various closures. A lot of studies have been devoted to the Doi-type kinetic theory and its approximation with various closures based on the Maier-Saupe potential and the Onsager potential (for example, Refs. [3-5, 8-17, 19, 23-28, 30, 35-38, 41, 43-49]). However, almost nothing can be found in the literature for the theoretical analysis of the Doi-Hess kinetic model with the Onsager interaction potential.

Recently the authors carried out a numerical study on the equilibrium states of rigid polymer rods with the Onsager potential. ${ }^{40}$ The primary purpose of the current paper is to extend the work in Ref. [40] and conduct a theoretical study on the full Doi-Hess kinetic theory with the Onsager interaction potential. We confine our attention to the two-dimensional liquid crystal polymers which is physically motivated by monolayer films in microelectronics and optoelectronics. Rigid, rodlike liquid crystalline polymers have been of considerable interest in thin films because of their stability, nonlinear optical properties, and role as alignment layers for liquid crystal displays. ${ }^{29}$ Experiments have shown that the dynamic behavior of monolayer nematic polymers can be quantitatively captured by the simple molecular model of Doi and Hess for both extensional and simple shear flows. ${ }^{29,32}$ Numerical and theoretical studies on the two-dimensional liquid crystal polymers have also been conducted by many researchers. $1,2,7,18,21-23,26-28,30,31,39,42,44,47,49$ Our work extends these previous papers to the Doi-Hess theory with the Onsager intermolecular potential.

This paper proceeds as follows. First, the mathematical framework of the two-dimensional Doi-Hess model with the Onsager potential is described in Section 2, and the connection between the Onsager potential and the Maier-Saupe potential is briefly discussed. In Section 3, an asymptotic analysis is carried out to calculate the nematic branches of polymers with the Onsager potential. It turns out that there are infinitely many nematic branches for polymers with the Onsager potential. A spectrum method is described in Section 4. The spectrum method is capable of reducing numerical errors to the computer precision. Numerical results obtained with the spectrum method are treated as the exact solution and are used to confirm the asymptotic results. In Section 5, the stability of the nematic branches is analyzed using free energy calculations. Finally, in Section 6, we summarize the results obtained in this paper.

\section{MATHEMATICAL FORMULATION OF THE TWO DIMENSIONAL DOI-HESS KINETIC MODEL WITH THE ONSAGER POTENTIAL}

We briefly review the two-dimensional mathematical formulation of the Doi-Hess kinetic theory for rigid rodlike nematogenic molecules immersed in a viscous solvent. ${ }^{6,20}$ In this theory, one considers a test molecule in a sea of others and models the interactions between molecules by a mean-field potential. The ensemble of polymer rods is described by an orientation probability density function (PDF). In the absence of flow, the orientation probability density function is governed by the Smoluchowski equation:

$$
\frac{\partial \rho}{\partial t}=D \frac{\partial}{\partial \mathbf{u}} \cdot\left(\frac{1}{k_{\mathrm{B}} T} \frac{\partial V}{\partial \mathbf{u}} \rho+\frac{\partial \rho}{\partial \mathbf{u}}\right)
$$

where $\rho(\mathbf{u}, t)$ is the probability density function that a polymer rod has orientation $\mathbf{u}$ at time $t, \mathbf{u}$ is a unit vector denoting the polymer rod orientation, $\partial / \partial \mathbf{u}$ is the gradient operator on the unit sphere, ${ }^{6} D$ the rotational diffusivity constant, $k_{\mathrm{B}}$ the Boltzmann constant, $T$ the absolute temperature and $V$ the mean-field potential representing the interactions between polymer rods.

In the two-dimensional space, the orientation of a polymer rod can be described by an angle $\theta$. The orientational vector of a polymer rod is given by $\mathbf{u}=(\cos \theta, \sin \theta)$. The Onsager intermolecular potential describes the interaction between the test polymer rod and all other polymer rods. The Onsager potential has the form

$$
U(\theta) \equiv \frac{V_{\text {Onsager }}}{k_{\mathrm{B}} T}=b \int_{0}^{2 \pi}|\sin (\tilde{\theta}-\theta)| \rho(\tilde{\theta}) d \tilde{\theta}
$$

where $b$ is the strength of the Onsager potential which is proportional to the normalized polymer concentration and is inversely proportional to the absolute temperature, and $\rho(\theta)$ the probability density function of the orientation angle $\theta$. For the convenience of discussions below, we use $U(\theta)$ to denote the Onsager potential normalized by the factor $k_{\mathrm{B}} T$. It should be pointed out that the Maier-Saupe intermolecular potential has a similar form as the Onsager potential with the kernel $|\sin (\tilde{\theta}-\theta)|$ in (2) being replaced by $|\sin (\tilde{\theta}-\theta)|^{2}=(1-\cos 2(\tilde{\theta}-\theta)) / 2$. The Maier-Saupe potential has the form

$$
\begin{aligned}
U(\theta) \equiv & \frac{V_{M S}}{k_{\mathrm{B}} T}=b \int_{0}^{2 \pi} \frac{1-\cos 2(\tilde{\theta}-\theta)}{2} \rho(\tilde{\theta}) d \tilde{\theta} \\
= & \frac{b}{2}-\frac{b}{2}\left[\cos 2 \theta \int_{0}^{2 \pi} \cos 2 \tilde{\theta} \rho(\tilde{\theta}) d \tilde{\theta}\right. \\
& \left.+\sin 2 \theta \int_{0}^{2 \pi} \sin 2 \tilde{\theta} \rho(\tilde{\theta}) d \tilde{\theta}\right]
\end{aligned}
$$

In the Maier-Saupe potential (3), the effect of $\rho(\tilde{\theta})$ is completely specified by two quantities $\int_{0}^{2 \pi} \cos 2 \tilde{\theta} \rho(\tilde{\theta}) d \tilde{\theta}$ and 
$\int_{0}^{2 \pi} \sin 2 \tilde{\theta} \rho(\tilde{\theta}) d \tilde{\theta}$. In other words, if these two quantities are given, then the Maier-Saupe potential is completely determined without knowing the probability density $\rho(\tilde{\theta})$. In contrast, the Onsager potential (2) depends on the whole function of the probability density $\rho(\tilde{\theta})$. This property of the Onsager potential causes significant mathematical difficulties for theoretical analysis.

In the current study of the two dimensional Doi-Hess kinetic model, we take the advantage that the probability density $\rho(\theta)$ is a periodic function. We consider the Fourier series expansion of the probability density function $\rho(\theta)$ :

$$
\rho(\theta)=\frac{1}{2 \pi}+\sum_{k=1}^{\infty}\left(a_{k} \cos k \theta+b_{k} \sin k \theta\right)
$$

where the constant term being $1 /(2 \pi)$ is dictated by the fact that the total probability is one: $\int_{0}^{2 \pi} \rho(\theta) d \theta=1$. Below, we will use the Fourier series of $\rho(\theta)$ to calculate the Fourier expansion of the normalized Onsager potential $U(\theta)$. We start with some mathematical preparations:

$$
\begin{aligned}
& \int_{0}^{2 \pi}|\sin \theta| \cos k \theta d \theta \\
& =\int_{0}^{\pi} \sin \theta \cos k \theta d \theta-\int_{\pi}^{2 \pi} \sin \theta \cos k \theta d \theta \\
& =\int_{0}^{\pi} \sin \theta \cos k \theta d \theta-\int_{0}^{\pi} \sin (2 \pi-\theta) \cos (2 k \pi-k \theta) d \theta \\
& =2 \int_{0}^{\pi} \sin \theta \cos k \theta d \theta \\
& =\left\{\begin{array}{l}
\frac{2}{k+1}-\frac{2}{k-1}=-\frac{4}{k^{2}-1}, \quad \text { for } k \text { even } \\
0, \quad \text { for } k \text { odd }
\end{array}\right. \\
& \int_{0}^{2 \pi}|\sin \theta| \sin k \theta d \theta=\int_{0}^{\pi} \sin \theta \sin k \theta d \theta-\int_{\pi}^{2 \pi} \sin \theta \sin k \theta d \theta \\
& =\int_{0}^{\pi} \sin \theta \sin k \theta d \theta \\
& \quad-\int_{0}^{\pi} \sin (2 \pi-\theta) \sin (2 k \pi-k \theta) d \theta=0
\end{aligned}
$$

With the help of (4), (5) and (6), we write the normalized Onsager potential as

$$
\begin{aligned}
U(\theta) & =b \int_{0}^{2 \pi}|\sin (\tilde{\theta}-\theta)| \rho(\tilde{\theta}) d \tilde{\theta} \\
& =b \int_{0-\theta}^{2 \pi-\theta}|\sin \tilde{\theta}| \rho(\tilde{\theta}+\theta) d \tilde{\theta} \\
& =b \int_{0}^{2 \pi}|\sin \tilde{\theta}| \rho(\tilde{\theta}+\theta) d \tilde{\theta} \\
& =b \int_{0}^{2 \pi}|\sin \tilde{\theta}|\left[\frac{1}{2 \pi}+\sum_{k=1}^{\infty}\left(a_{k} \cos k(\tilde{\theta}+\theta)\right.\right. \\
& \left.\left.\quad+b_{k} \sin k(\tilde{\theta}+\theta)\right)\right] d \tilde{\theta}
\end{aligned}
$$

The equilibrium probability density of Smoluchowski equation (1) is given by the Boltzmann distribution:

$$
\begin{aligned}
\rho(\theta) & =\frac{1}{Z} \exp [-U(\theta)] \\
& =\frac{1}{Z} \exp \left(\sum_{j=1}^{\infty}\left(c_{2 j} \cos 2 j \theta+d_{2 j} \sin 2 j \theta\right)\right)
\end{aligned}
$$

where $Z$ is the partition function given by

$$
\begin{aligned}
Z & =\int_{0}^{2 \pi} \exp [-U(\theta)] d \theta \\
& =\int_{0}^{2 \pi} \exp \left(\sum_{j=1}^{\infty}\left(c_{2 j} \cos 2 j \theta+d_{2 j} \sin 2 j \theta\right)\right) d \theta
\end{aligned}
$$

Recall that we started with the Fourier series expansion of $\rho(\theta)$ with coefficients $a_{j}$ and $b_{j}$. The Fourier coefficients with even indices, $a_{2 k}$ and $b_{2 k}$, can be expressed in terms of $c_{2 k}$ and $d_{2 k}$ from (8).

$$
a_{2 k}=\frac{1}{4 b}\left[(2 k)^{2}-1\right] c_{2 k}, \quad b_{2 k}=\frac{1}{4 b}\left[(2 k)^{2}-1\right] d_{2 k}
$$

Below we use (10) to show that the Fourier coefficients of $\rho(\theta)$ with odd indices are zero. The probability density $\rho(\theta)$ given in (10) satisfies

$$
\rho(\theta+\pi)=\rho(\theta)
$$


With the help of (13), we have

$$
\begin{aligned}
a_{2 k+1}= & \frac{1}{\pi} \int_{0}^{2 \pi} \cos [(2 k+1) \theta] \rho(\theta) d \theta \\
= & \frac{1}{\pi} \int_{0}^{\pi} \cos [(2 k+1) \theta] \rho(\theta) d \theta \\
& \quad+\frac{1}{\pi} \int_{\pi}^{2 \pi} \cos [(2 k+1) \theta] \rho(\theta) d \theta \\
= & \frac{1}{\pi} \int_{0}^{\pi} \cos [(2 k+1) \theta] \rho(\theta) d \theta \\
& \quad+\frac{1}{\pi} \int_{0}^{\pi} \cos [(2 k+1) \theta+\pi] \rho(\theta+\pi) d \theta=0
\end{aligned}
$$

Similarly, one can verify that

$$
b_{2 k+1}=\frac{1}{\pi} \int_{0}^{2 \pi} \sin [(2 k+1) \theta] \rho(\theta) d \theta=0
$$

Next we show that $U(\theta)$ can be made an even function of $\theta$ by shifting the coordinate system. That is, we can make $b_{2 k}=0$ for all $k$ by a single shifting. We start by using relation (12) to express function $\rho(\theta)$ in terms of function $U(\theta)$ and its derivatives.

$$
\rho(\theta)=\frac{1}{2 \pi}+\frac{1}{4 b}\left[U^{\prime \prime}(\theta)+U(\theta)\right]
$$

The equilibrium probability density function $\rho(\theta)$ is also linked to the normalized Onsager potential $U(\theta)$ through the Boltzmann relation

$$
\rho(\theta)=\frac{1}{Z} \exp [-U(\theta)]
$$

Combining (16) and (17), we obtain a differential equation for $U(\theta)$.

$$
U^{\prime \prime}(\theta)=-U(\theta)+\frac{4 b}{Z} \exp [-U(\theta)]-\frac{4 b}{2 \pi}
$$

(18) is a second order differential equation. To determine the solution of a second order differential equation, we need to specify two initial conditions. Let $\theta_{0}$ be the location at which $\rho(\theta)$ attains its global maximum. It follows immediately from (17) that the normalized Onsager potential $U(\theta)$ attains its global minimum at $\theta_{0}$. Without loss of generality, we assume $\theta_{0}=0$ (one can always shift the coordinate system to make $\theta_{0}=0$ ). At the location of the global maximum, we have

$$
U(0)=U_{0}, \quad U^{\prime}(0)=0
$$

We use (19) as the initial conditions for differential equation (18). It is straightforward to verify that both differential equation (18) and initial conditions (19) are invariant under the change of variable $\theta \rightarrow-\theta$. Therefore, we conclude that $U(\theta)$ is an even function

$$
U(\theta)=U(-\theta)
$$

Consequently, from (9) we have $d_{2 k}=0$ and $U(\theta)$ is simplified to

$$
U(\theta)=-\sum_{k=1}^{\infty} c_{2 k} \cos 2 k \theta
$$

The next task is to derive a (nonlinear) system for Fourier coefficients $c_{2 k}$. Relation (12) provides one set of equations for Fourier coefficients $a_{2 k}$ and $c_{2 k}$. To derive another set of equations for $a_{2 k}$ and $c_{2 k}$, we calculate the Fourier coefficients of $\rho(\theta)$ using the expression of $\rho(\theta)$ given in (10)

$$
\begin{aligned}
a_{2 k} & =\frac{1}{\pi} \int_{0}^{2 \pi} \cos 2 k \theta \rho(\theta) d \theta \\
& =\frac{1}{\pi} \cdot \frac{\int_{0}^{2 \pi} \cos 2 k \theta \exp [-U(\theta)] d \theta}{\int_{0}^{2 \pi} \exp [-U(\theta)] d \theta} \\
& =\frac{1}{\pi} \cdot \frac{\int_{0}^{2 \pi} \cos 2 k \theta \exp \left[\sum_{i=1}^{\infty} c_{2 i} \cos 2 i \theta\right] d \theta}{\int_{0}^{2 \pi} \exp \left[\sum_{i=1}^{\infty} c_{2 i} \cos 2 i \theta\right] d \theta}
\end{aligned}
$$

Combining (12) and (22), we arrive at a nonlinear system for coefficients $\left\{c_{2 k}\right\}$.

$$
\frac{\int_{0}^{2 \pi} \cos 2 k \theta \exp \left[\sum_{i=1}^{\infty} c_{2 i} \cos 2 i \theta\right] d \theta}{\int_{0}^{2 \pi} \exp \left[\sum_{i=1}^{\infty} c_{2 i} \cos 2 i \theta\right] d \theta}=\frac{\pi}{4 b}\left[(2 k)^{2}-1\right] c_{2 k}
$$

Since the Boltzmann relation establishes a one-to-one correspondence between the probability density $\rho(\theta)$ and the Onsager potential $U(\theta)$ (if we set the constant term to zero in the Fourier expansion of the Onsager potential), we only need to solve for the Onsager potential. In a properly selected coordinate system, the Onsager potential is an even function and its Fourier coefficients satisfy $c_{2 k+1}=0$ and satisfy nonlinear system (23). The nonlinear system (23) is the key governing equation in our study. Below we will solve it first asymptotically and then numerically with a very accurate numerical method.

\section{PHASE DIAGRAM OF NEMATIC POLYMERS WITH THE ONSAGER POTENTIAL}

We now solve nonlinear system (23) to obtain the phase diagram of nematic polymers with the Onsager interaction potential. We carry out asymptotic analysis for the case where the amplitude of the normalized Onsager potential is small. Since the probability density and the Onsager potential are related by the Boltzmann distribution, small amplitude in the Onsager potential implies that the probability density is not far from being a constant. That is, we do asymptotic analysis on the nematic states that are close to the isotropic state. Mathematically, we assume the Fourier coefficients of the Onsager potential have the expansions

$$
c_{2 j}=\varepsilon \alpha_{2 j}+\varepsilon^{2} \beta_{2 j}+O\left(\varepsilon^{3}\right)
$$

where $\varepsilon$ is a small parameter, and $\alpha_{2 j}$ and $\beta_{2 j}$ are coefficients independent of $\varepsilon$. At this point, the meaning of $\varepsilon$ 
is not precisely defined yet. Later we will re-define $\varepsilon$ precisely. Expanding the numerator and denominator on the left side of (23) in powers of $\varepsilon$ leads to

$$
\begin{gathered}
\int_{0}^{2 \pi} \cos 2 k \theta \exp \left[\sum_{i=1}^{\infty}\left(\varepsilon \alpha_{2 i}+\varepsilon^{2} \beta_{2 i}\right) \cos 2 i \theta\right] d \theta \\
=\int_{0}^{2 \pi} \cos 2 k \theta\left[1+\sum_{i=1}^{\infty}\left(\varepsilon \alpha_{2 i}+\varepsilon^{2} \beta_{2 i}\right) \cos 2 i \theta\right. \\
\left.\quad+\frac{1}{2} \varepsilon^{2}\left(\sum_{i=1}^{\infty} \alpha_{2 i} \cos 2 i \theta\right)^{2}+O\left(\varepsilon^{3}\right)\right] d \theta \\
=\left(\varepsilon \alpha_{2 k}+\varepsilon^{2} \beta_{2 k}\right) \pi \\
+\frac{1}{2} \varepsilon^{2} \int_{0}^{2 \pi} \cos 2 k \theta\left(\sum_{i=1}^{\infty} \alpha_{2 i} \cos 2 i \theta\right)^{2} d \theta+O\left(\varepsilon^{3}\right)
\end{gathered}
$$

and

$$
\begin{aligned}
\int_{0}^{2 \pi} \exp \left[\sum_{i=1}^{\infty}\left(\varepsilon \alpha_{2 i}+\varepsilon^{2} \beta_{2 i}\right) \cos 2 i \theta\right] d \theta \\
=\int_{0}^{2 \pi}\left[1+\sum_{i=1}^{\infty}\left(\varepsilon \alpha_{2 i}+\varepsilon^{2} \beta_{2 i}\right) \cos 2 i \theta+O\left(\varepsilon^{2}\right)\right] d \theta \\
=2 \pi\left[1+O\left(\varepsilon^{2}\right)\right]
\end{aligned}
$$

respectively. In general, we expect the Onsager potential $U(\theta)$ changes with the normalized polymer concentration $b$, which suggests that $b$ is not independent of $\varepsilon$. We assume $b$ has the expansion

$$
b=b^{(0)}\left[1+\varepsilon b^{(1)}+O\left(\varepsilon^{2}\right)\right]
$$

Substituting these results into Eq. (23), and first looking at only the coefficients of the first order $(O(\varepsilon))$ terms, we obtain

$$
\varepsilon \frac{\alpha_{2 k}}{2}=\varepsilon \frac{\pi}{4 b^{(0)}}\left[(2 k)^{2}-1\right] \alpha_{2 k}
$$

which in turn yields

$$
\alpha_{2 k}\left\{1-\frac{\pi}{2 b^{(0)}}\left[(2 k)^{2}-1\right]\right\}=0
$$

Below we consider possible solutions of (29) in several cases.

- Case 1: If $\alpha_{2} \neq 0$, then in (29) for $k=1$ we must have

$$
1-\frac{\pi}{2 b^{(0)}}\left[2^{2}-1\right]=0
$$

which yields $b^{(0)}=\left(\left(2^{2}-1\right) / 2\right) \pi=(3 / 2) \pi$. Consequently, for $k>1$, we have $1-\left(\pi / 2 b^{(0)}\right)\left[(2 k)^{2}-1\right]=$ $\left(2^{2}-(2 k)^{2}\right) /\left(2^{2}-1\right)<0$. It follows from (29) that $\alpha_{2 k}=0$ for $k>1$. Recall that the meaning of $\varepsilon$ in the expansion is not precisely defined yet. When $\alpha_{2} \neq 0$, we re-define $c_{2}=$ $\alpha_{2} \varepsilon+\beta_{2} \varepsilon^{2}+O\left(\varepsilon^{3}\right)$ as the new $\varepsilon$, which is equivalent to setting $\alpha_{2}=1$ and $\beta_{2}=0$. Substituting $\alpha_{2}=1$ and $\alpha_{2 k}=0$ for $k>1$ into (25), we have

RHS of (25)

$$
=\left(\varepsilon \alpha_{2 k}+\varepsilon^{2} \beta_{2 k}\right) \pi+\left\{\begin{array}{ll}
\varepsilon^{2} \frac{\pi}{4}, & k=2 \\
0, & \text { otherwise }
\end{array}+O\left(\varepsilon^{3}\right)\right.
$$

In the calculation of coefficients $\alpha_{2 k}$ above, we keep only the first order terms in Eq. (23). Now in the calculation of coefficients $\beta_{2 k}$, we keep both the first order and the second order terms. We first calculate $b^{(1)}$. For $k=1$, Eq. (23) becomes

$$
\frac{\varepsilon+O\left(\varepsilon^{3}\right)}{1+O\left(\varepsilon^{2}\right)}=\frac{\varepsilon}{1+\varepsilon b^{(1)}+O\left(\varepsilon^{2}\right)}
$$

which yields $b^{(1)}=0$. For $k=2,(23)$ provides us an equation for $\beta_{4}$ :

$$
\frac{\varepsilon^{2} \beta_{4}+\varepsilon^{2} 1 / 4+O\left(\varepsilon^{3}\right)}{1+O\left(\varepsilon^{2}\right)}=\frac{4^{2}-1}{2^{2}-1} \cdot \frac{\varepsilon^{2} \beta_{4}}{1+O\left(\varepsilon^{2}\right)}
$$

which leads to $\beta_{4}=1 / 16$. For $k>2$, Eq. (23) takes the form

$$
\frac{\varepsilon^{2} \beta_{2 k}+O\left(\varepsilon^{3}\right)}{1+O\left(\varepsilon^{2}\right)}=\frac{(2 k)^{2}-1}{3} \cdot \frac{\varepsilon^{2} \beta_{2 k}}{1+O\left(\varepsilon^{2}\right)}
$$

which gives us $\beta_{2 k}=0$ for $k>2$. Therefore, the nematic state that has a small non-zero coefficient for $\cos 2 \theta$ in the Fourier expansion of $U(\theta)$ is described by

$$
\begin{gathered}
U(\theta)=-\varepsilon \cos 2 \theta-\frac{\varepsilon^{2}}{16} \cos 4 \theta+O\left(\varepsilon^{3}\right) \\
b=\frac{3}{2} \pi\left(1+O\left(\varepsilon^{2}\right)\right)
\end{gathered}
$$

To calculate the coefficient of the second order term in the expansion of $b$, we keep one more term in the expansions of (25) and (26):

$$
\begin{gathered}
\text { RHS of }(25)=\varepsilon \pi+\varepsilon^{3} \frac{5 \pi}{32}+O\left(\varepsilon^{4}\right), \quad k=1 \\
\text { RHS of }(26)=2 \pi\left(1+\frac{1}{4} \varepsilon^{2}+O\left(\varepsilon^{3}\right)\right)
\end{gathered}
$$

An equation for $b^{(2)}$ can be derived by substituting results (32) and (33) into Eq. (23) for $k=1$ :

$$
\frac{\varepsilon+5 / 32 \varepsilon^{3}+O\left(\varepsilon^{4}\right)}{1+1 / 4 \varepsilon^{2}+O\left(\varepsilon^{3}\right)}=\frac{\varepsilon}{1+b^{(2)} \varepsilon^{2}+O\left(\varepsilon^{3}\right)}
$$

which gives us $b^{(2)}=3 / 32$ and thus, we obtain

$$
b=\frac{3}{2} \pi\left(1+\frac{3}{32} \varepsilon^{2}+O\left(\varepsilon^{4}\right)\right)
$$


It is desirable to express the normalized Onsager potential $U(\theta)$ as a function of the normalized polymer concentration $b$. For that purpose, we solve for $\varepsilon$ from (34) and arrive at

$$
\begin{gathered}
\varepsilon= \pm \sqrt{\frac{b-(3 / 2) \pi}{(9 / 64) \pi}} \\
U(\theta)=-\varepsilon \cos 2 \theta-\frac{\varepsilon^{2}}{16} \cos 4 \theta+O\left(\varepsilon^{3}\right)
\end{gathered}
$$

where the sign \pm means that there are two equivalent branches of nematic state near $b=(3 / 2) \pi$. These two branches are equivalent to each other via a 90 degree rotation. The equilibrium probability density is given by

$$
\rho(\theta)=\frac{1}{2 \pi}+\varepsilon \frac{3}{4 b} \cos 2 \theta+\varepsilon^{2} \frac{15}{64 b} \cos 4 \theta+O\left(\varepsilon^{3}\right)
$$

where $\varepsilon$ is given in (35). This is the first nematic branch of polymer ensembles with the Onsager potential. Before we plot the first nematic branch, we need to decide on how to represent the nematic state as a function of $b$. We could plot coefficients $\left(c_{2}, c_{4}, \ldots, c_{2 j}, \ldots\right)$ as functions of $b$. But that plot is a curve in a space of infinite dimension, which is difficult to illustrate. To represent the phase diagram using a simple two-dimensional plot, we plot the leading nonzero coefficient in $\left(c_{2}, c_{4}, \ldots, c_{2 j}, \ldots\right)$. This approach of plotting the leading non-zero Fourier coefficients is in, fact, consistent with what has been done in the case of nematic polymers with the Maier-Saupe interaction potential. For the Maier-Saupe potential, $c_{2}$ is the only possible nonzero Fourier coefficient and other Fourier coefficients are always zero because by definition the Maier-Saupe potential contains only the $\cos 2 \theta$ term. In contrast, in general the Onsager potential contains all $\cos 2 j \theta$ terms where $j$ goes from 1 to infinity. For the onsager potential, any member in set $\left(c_{2}, c_{4}, \ldots, c_{2 j}, \ldots\right)$ can be the leading non-zero coefficient. For the first nematic branch of polymer ensembles with the Onsager potential, $c_{2}$ is the leading non-zero coefficient. The behavior of this nematic branch is shown in Figure 1.

In Figure 1, the vertical coordinate $c_{2}$ is a measure of the magnitude of the Onsager potential for the first nematic branch. The equilibrium probability density is related to the Onsager potential by the Boltzmann distribution. Thus, $c_{2}$ also measures how far the nematic state deviates from the isotropic state. In this sense, $c_{2}$ can be viewed as an unnormalized order parameter for the first nematic branch. In Figure 1, the solid line represents a very accurate numerical solution, which is treated as the exact solution, and the dashed line represents the asymptotic solution obtained above. The numerical method will be discussed in the next section.

For polymer ensembles with the Maier-Saupe interaction potential, the polymer orientation distribution is often illustrated by plotting in polar coordinate system a function $f(\theta)$ showing the alignment of polymer rods along the

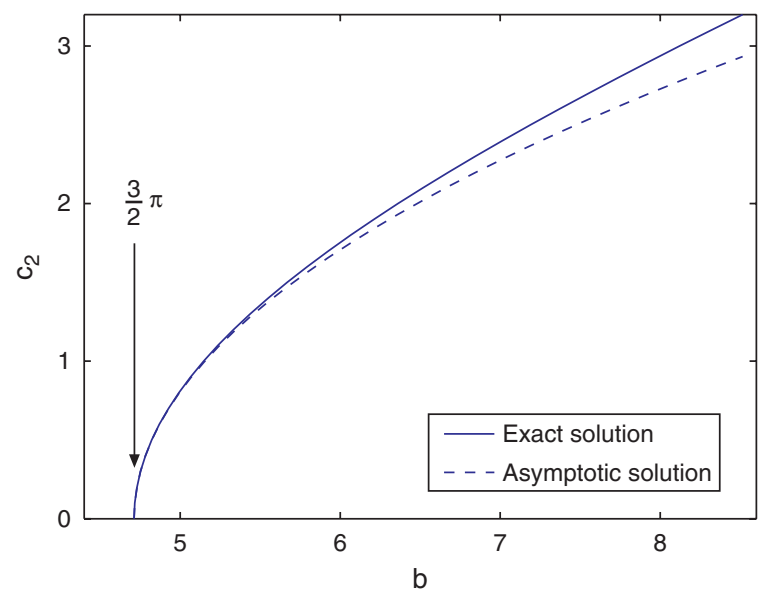

Fig. 1. The first nematic branch of polymer ensemble with the Onsager interaction potential. This nematic branch intersects with the isotropic branch, at $(3 / 2) \pi$. The horizontal coordinate $b$ is the normalized polymer concentration; the vertical coordinate $c_{2}$ is the coefficient of the $\cos 2 \theta$ term (the leading non-zero term for the first nematic branch) in the Fourier expansion of the normalized Onsager potential.

direction with angle $\theta$. Function $f(\theta)$ is defined as the rootmean-square of the inner-product between polymer rods in the ensemble and the direction $\theta$.

$$
\begin{aligned}
f(\theta) & =\sqrt{\int_{0}^{2 \pi}((\cos \theta, \sin \theta) \cdot(\cos \tilde{\theta}, \sin \tilde{\theta}))^{2} \rho(\tilde{\theta}) d \tilde{\theta}} \\
& =\sqrt{\int_{0}^{2 \pi} \cos ^{2}(\theta-\tilde{\theta}) \rho(\tilde{\theta}) d \tilde{\theta}} \\
& =\sqrt{\int_{0}^{2 \pi} \frac{1+\cos 2(\theta-\tilde{\theta})}{2} \rho(\tilde{\theta}) d \tilde{\theta}} \\
& =\sqrt{\frac{1}{2}+\frac{1}{2} \cos 2 \theta \int_{0}^{2 \pi} \cos 2 \tilde{\theta} \rho(\tilde{\theta}) d \tilde{\theta}} \\
& =\sqrt{\frac{1}{2}+\frac{1}{2} a_{2} \pi \cos 2 \theta}=\sqrt{\frac{1}{2}+\frac{3 \pi}{8 b} c_{2} \cos 2 \theta}
\end{aligned}
$$

In Figure 2, we plot in polar coordinate system probability density $\rho(\theta)$ and function $f(\theta)$ of a typical equilibrium state on the first nematic branch. Note that in some literature, the graph of function $f(\theta)$ was mistakenly identified as an ellipse. This misconception was probably caused by the fact that the function form of $f^{2}(\theta)$, when written in terms of the Cartesian coordinates $(x, y)$, has an elliptic form. Using $x=\cos \theta$ and $y=\sin \theta$, we have

$$
\begin{aligned}
f^{2}(x, y) & =\frac{1}{2}+\frac{3 \pi}{8 b} c_{2} \cos 2 \theta \\
& =\left(\frac{1}{2}+\frac{3 \pi}{8 b} c_{2}\right) \cos ^{2} \theta+\left(\frac{1}{2}-\frac{3 \pi}{8 b} c_{2}\right) \sin ^{2} \theta \\
& =\left(\frac{1}{2}+\frac{3 \pi}{8 b} c_{2}\right) x^{2}+\left(\frac{1}{2}-\frac{3 \pi}{8 b} c_{2}\right) y^{2}
\end{aligned}
$$



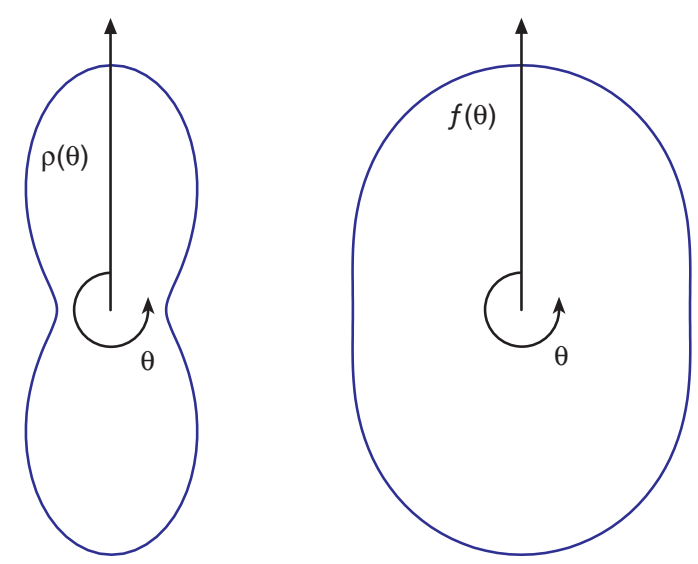

Fig. 2. Probability density $\rho(\theta)$ and function $f(\theta)$ shown in polar coordinate system for a typical equilibrium state on the first nematic branch.

It can be shown that the coefficient $\left(1 / 2-(3 \pi /(8 b)) c_{2}\right.$ is always positive. So indeed $f^{2}(x, y)$ has an elliptic form. But the graph of $f(x, y)$ with the constraint $x^{2}+y^{2}=1$ is not exactly an ellipse although it looks not very different from an ellipse.

In Figure 2, the graph of probability density $\rho(\theta)$ is significantly different from an ellipse. It has a dumbbell shape. Actually, the graph of probability density $\rho(\theta)$ does not always have a dumbbell shape. For small value of $c_{2}$, that is, for $b$ sufficiently close to $(3 / 2) \pi$, the graph of $\rho(\theta)$ looks like an ellipse in the sense that it is convex everywhere. In particular, it is convex at $\theta=(1 / 2) \pi$. The curvature of $\rho(\theta)$ is given by

$$
\kappa(\theta)=\frac{\rho^{2}(\theta)+2\left(\rho^{\prime}(\theta)\right)^{2}-\rho(\theta) \rho^{\prime \prime}(\theta)}{\left(\rho^{2}(\theta)+\left(\rho^{\prime}(\theta)\right)^{2}\right)^{3 / 2}}
$$

At $\theta=(1 / 2) \pi$ and for small value of $c_{2}=\varepsilon$, we have

$$
\begin{gathered}
\rho\left(\frac{1}{2} \pi\right)=\frac{1}{2 \pi}-\frac{1}{2 \pi} \varepsilon+O\left(\varepsilon^{2}\right) \\
\rho^{\prime}\left(\frac{1}{2} \pi\right)=0 \\
\rho^{\prime \prime}\left(\frac{1}{2} \pi\right)=\frac{1}{2 \pi} 4 \varepsilon+O\left(\varepsilon^{2}\right) \\
\kappa\left(\frac{1}{2} \pi\right)=\frac{1}{\rho((1 / 2) \pi)}(1-5 \varepsilon)+O\left(\varepsilon^{2}\right)
\end{gathered}
$$

Thus, for small values of $c_{2}=\varepsilon$, the curvature $\kappa((1 / 2) \pi)$ is positive and the graph of $\rho(\theta)$ is convex. The results shown in Figure 1 demonstrate that the asymptotic solution is still valid even for fairly large $\varepsilon$. Suppose the asymptotic expression of $\kappa((1 / 2) \pi)$ given in (41) is valid for moderately large $\varepsilon$. We can use the asymptotic formula to determine at what value of $\varepsilon$ the curvature changes sign from positive to negative. Setting $\kappa((1 / 2) \pi)=0$, we obtain $\varepsilon=0.2$, which is approximately the value at which $\rho(\theta)$ change from being convex to being concave at $\theta=(1 / 2) \pi$. This has been confirmed in our numerical simulations. Once $\rho(\theta)$ becomes concave at $\theta=(1 / 2) \pi$, the graph of $\rho(\theta)$ takes the shape of a dumbbell.

- Case 2: If $\alpha_{2}=0$ but $\alpha_{4} \neq 0$, then in (29) for $k=2$ we must have

$$
1-\frac{\pi}{2 b^{(0)}}\left[4^{2}-1\right]=0
$$

which yields $b^{(0)}=\left(4^{2}-1\right) / 2 \pi=(15 / 2) \pi$. Consequently, for $k>2$, we have $1-\pi /\left(2 b^{(0)}\right)\left[(2 k)^{2}-1\right]=$ $\left(4^{2}-(2 k)^{2}\right) /\left(4^{2}-1\right)<0$. It follows from (29) that $\alpha_{2 k}=0$ for $k>2$. As we did in case 1 , when $\alpha_{4} \neq 0$, we re-define $c_{4}=\alpha_{4} \varepsilon+\beta_{4} \varepsilon^{2}+O\left(\varepsilon^{3}\right)$ as the new $\varepsilon$, which is equivalent to setting $\alpha_{4}=1$ and $\beta_{4}=0$. Substituting $\alpha_{4}=1$ and $\alpha_{2 k}=0$ for $k \neq 2$ into (25), we have

RHS of (25)

$$
=\left(\varepsilon \alpha_{2 k}+\varepsilon^{2} \beta_{2 k}\right) \pi+\left\{\begin{array}{ll}
\varepsilon^{2} \frac{\pi}{4}, & k=4 \\
0, & \text { otherwise }
\end{array}+O\left(\varepsilon^{3}\right)\right.
$$

As in case 1 , once we finish calculating coefficients $\alpha_{2 k}$, we retain both the first order terms and the second order terms in Eq. (23) to calculate coefficients $\beta_{2 k}$. We first calculate $b^{(1)}$. For $k=2$, Eq. (23) simply can be written as

$$
\frac{\varepsilon+O\left(\varepsilon^{3}\right)}{1+O\left(\varepsilon^{2}\right)}=\frac{\varepsilon+O\left(\varepsilon^{3}\right)}{1+\varepsilon b^{(1)}}
$$

which immediately implies $b^{(1)}=0$. For $k=4$, (23) gives an equation for $\beta_{8}$ :

$$
\frac{\varepsilon^{2} \beta_{8}+\varepsilon^{2}(1 / 4)+O\left(\varepsilon^{3}\right)}{1+O\left(\varepsilon^{2}\right)}=\frac{8^{2}-1}{4^{2}-1} \cdot \frac{\varepsilon^{2} \beta_{8}}{1+O\left(\varepsilon^{2}\right)}
$$

which yields $\beta_{8}=(5 / 64)$. For $k \notin\{2,4\}$, Eq. (23) takes the form

$$
\frac{\varepsilon^{2} \beta_{2 k}+O\left(\varepsilon^{3}\right)}{1+O\left(\varepsilon^{2}\right)}=\frac{(2 k)^{2}-1}{4^{2}-1} \cdot \frac{\varepsilon^{2} \beta_{2 k}}{1+O\left(\varepsilon^{2}\right)}
$$

which, along with $\beta_{4}=0$, gives us $\beta_{2 k}=0$ for $k \neq 4$. Therefore, the nematic state, which has zero coefficient for $\cos 2 \theta$ but has a small non-zero coefficient for $\cos 4 \theta$ in the Fourier expansion of $U(\theta)$, is given by

$$
\begin{gathered}
U(\theta)=-\varepsilon \cos 4 \theta-\frac{5}{64} \varepsilon^{2} \cos 8 \theta+O\left(\varepsilon^{3}\right) \\
b=\frac{15}{2} \pi\left(1+O\left(\varepsilon^{2}\right)\right)
\end{gathered}
$$

To calculate the coefficient of the second order term in the expansion of $b$, we retain one more term in the expansions of (25) and (26)

$$
\begin{gathered}
\text { RHS of }(25)=\varepsilon \pi+\varepsilon^{3} \frac{21 \pi}{128}+O\left(\varepsilon^{4}\right), \quad k=2 \\
\text { RHS of }(26)=2 \pi\left(1+\frac{1}{4} \varepsilon^{2}+O\left(\varepsilon^{3}\right)\right)
\end{gathered}
$$


An equation for $b^{(2)}$ can be found by substituting results (44) and (45) into Eq. (23) for $k=2$ :

$$
\frac{\varepsilon+(21 / 128) \varepsilon^{3}+O\left(\varepsilon^{4}\right)}{1+(1 / 4) \varepsilon^{2}+O\left(\varepsilon^{3}\right)}=\frac{\varepsilon}{1+b^{(2)} \varepsilon^{2}+O\left(\varepsilon^{3}\right)}
$$

which gives $b^{(2)}=11 / 128$ and therefore, we obtain

$$
b=\frac{15}{2} \pi\left(1+\frac{11}{128} \varepsilon^{2}+O\left(\varepsilon^{4}\right)\right)
$$

In order to express the normalized Onsager potential $U(\theta)$ as a function of the normalized polymer concentration $b$, we solve for $\varepsilon$ from (46) and arrive at

$$
\begin{gathered}
\varepsilon= \pm \sqrt{\frac{b-(15 / 2) \pi}{(165 / 256) \pi}} \\
U(\theta)=-\varepsilon \cos 4 \theta-\frac{5}{64} \varepsilon^{2} \cos 8 \theta+O\left(\varepsilon^{3}\right)
\end{gathered}
$$

Again, the sign \pm indicates that there are two equivalent branches of nematic state near $b=(15 / 2) \pi$. These two branches are equivalent to each other via a 90 degree rotation. The equilibrium probability density can be written as

$\rho(\theta)=\frac{1}{2 \pi}+\varepsilon \frac{15}{4 b} \cos 4 \theta+\varepsilon^{2} \frac{315}{256 b} \varepsilon^{2} \cos 8 \theta+O\left(\varepsilon^{3}\right)$

where $\varepsilon$ is given in (47). This is the second nematic branch for polymer ensembles with the Onsager potential. The behavior of this nematic branch is shown in Figure 3.

In Figure 3, the vertical coordinate $c_{4}$ measures the magnitude of the Onsager potential for the second branch. The equilibrium probability density is related to the Onsager potential by the Boltzmann distribution. Thus, $c_{4}$ also

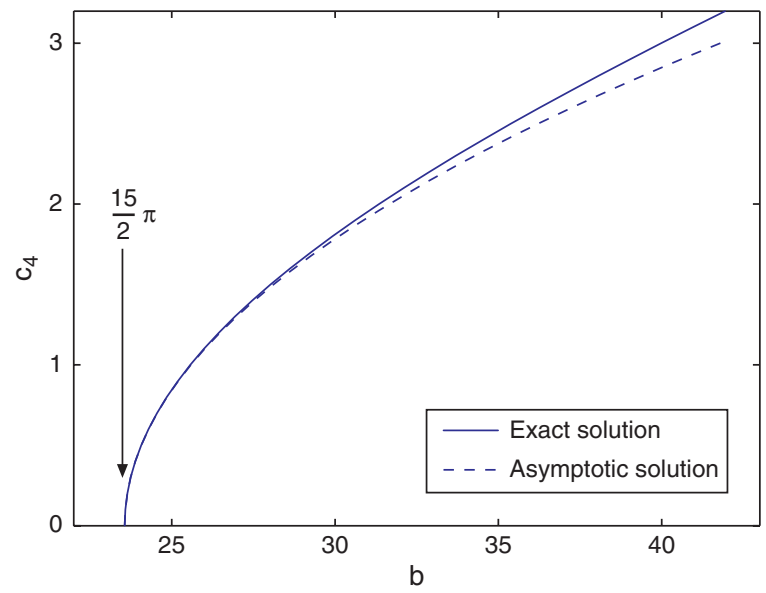

Fig. 3. The second nematic branch of polymer ensembles with the Onsager interaction potential. This nematic branch intersects with the isotropic branch at $(15 / 2) \pi$. Here the horizontal coordinate $b$ denotes the normalized polymer concentration whereas the vertical coordinate $c_{4}$ is the coefficient of the $\cos 4 \theta$ term (the leading non-zero term for the second nematic branch) in the Fourier expansion of the normalized Onsager potential. reflects how far the nematic state deviates from the isotropic state. In this sense, $c_{4}$ can be thought of as an unnormalized order parameter for the second branch. In Figure 3 , the solid line corresponds to a very accurate numerical solution, which is taken as the exact solution, and the dashed line denotes the asymptotic solution obtained above. Note that for the second nematic branch, the meaning of the order parameter is different from that of the first nematic branch. For the first nematic branch and for the case of the Maier-Saupe potential, the order parameter measures the alignment of polymer rods along one direction (the direction along which the alignment is maximized). For the second nematic polymer, as we will see below, the order parameter $c_{4}$ measures the alignment of polymer rods with two perpendicular directions.

Figure 4 depicts in polar coordinate system the probability density $\rho(\theta)$ of a typical equilibrium state on the second nematic branch. Function $f(\theta)$, the root-mean-square of the inner-product between polymer rods and the direction $\theta$, is not shown. In fact, for the second nematic branch, $c_{2}=0$ and $f(\theta)=$ const. More specifically,

$$
f(\theta)=\sqrt{\frac{1}{2}+\frac{3 \pi}{8 b} c_{2} \cos 2 \theta}=\sqrt{\frac{1}{2}}
$$

Figure 4 shows that the probability density $\rho(\theta)$ has four fold symmetry and the polymer rods are equally likely being aligned along the horizontal direction and being aligned along the vertical direction.

- Case 3: If $\alpha_{2}=\alpha_{4}=0$ but $\alpha_{6} \neq 0$, then in (29) for $k=3$ we must have

$$
1-\frac{\pi}{2 b^{(0)}}\left[6^{2}-1\right]=0
$$

which yields $b^{(0)}=\left(\left(6^{2}-1\right) / 2\right) \pi=(35 / 2) \pi$. Consequently, for $k>3$, we have $1-\left(\pi /\left(2 b^{(0)}\right)\right)\left[(2 k)^{2}-1\right]=$ $\left(6^{2}-(2 k)^{2}\right) /\left(6^{2}-1\right)<0$. It follows from (29) that $\alpha_{2 k}=0$ for $k>3$. Since $\alpha_{6} \neq 0$, we re-define $c_{6}=\alpha_{6} \varepsilon+\beta_{6} \varepsilon^{2}+$ $O\left(\varepsilon^{3}\right)$ as the new $\varepsilon$, which is equivalent to setting $\alpha_{6}=1$

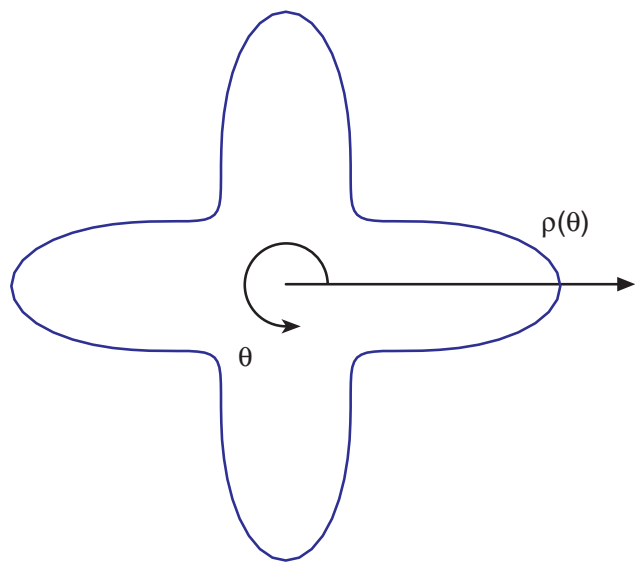

Fig. 4. Probability density $\rho(\theta)$ shown in polar coordinate system for a typical equilibrium state on the second nematic branch. 
and $\beta_{6}=0$. Substituting $\alpha_{6}=1$ and $\alpha_{2 k}=0$ for $k \neq 3$ into (25), we have

RHS of (25)

$$
=\left(\varepsilon \alpha_{2 k}+\varepsilon^{2} \beta_{2 k}\right) \pi+\left\{\begin{array}{ll}
\varepsilon^{2} \frac{\pi}{4}, & k=6 \\
0, & \text { otherwise }
\end{array}+O\left(\varepsilon^{3}\right)\right.
$$

Now we continue to calculate coefficients $\beta_{2 k}$. For that purpose, we keep both the first order terms and the second order terms in Eq. (23). We first calculate $b^{(1)}$. For $k=3$, Eq. (23) simplifies to

$$
\frac{\varepsilon+O\left(\varepsilon^{3}\right)}{1+O\left(\varepsilon^{2}\right)}=\frac{\varepsilon+O\left(\varepsilon^{3}\right)}{1+\varepsilon b^{(1)}}
$$

which gives $b^{(1)}=0$. For $k=6$, (23) yields an equation for $\beta_{12}$.

$$
\frac{\varepsilon^{2} \beta_{12}+\varepsilon^{2}(1 / 4)+O\left(\varepsilon^{3}\right)}{1+O\left(\varepsilon^{2}\right)}=\frac{12^{2}-1}{6^{2}-1} \cdot \frac{\varepsilon^{2} \beta_{12}}{1+O\left(\varepsilon^{2}\right)}
$$

which implies $\beta_{12}=35 / 432$. For $k \notin\{3,6\}$, Eq. (23) becomes

$$
\frac{\varepsilon^{2} \beta_{2 k}+O\left(\varepsilon^{3}\right)}{1+O\left(\varepsilon^{2}\right)}=\frac{(2 k)^{2}-1}{6^{2}-1} \cdot \frac{\varepsilon^{2} \beta_{2 k}}{1+O\left(\varepsilon^{2}\right)}
$$

which, along with $\beta_{6}=0$, yields $\beta_{2 k}=0$ for $k \neq 6$. Therefore, the nematic state that has zero coefficients for $\cos 2 \theta$ and for $\cos 4 \theta$ but has a small non-zero coefficient for $\cos 6 \theta$ in the Fourier expansion of $U(\theta)$ can be expressed as

$$
\begin{gathered}
U(\theta)=-\varepsilon \cos 6 \theta-\frac{35}{432} \varepsilon^{2} \cos 12 \theta+O\left(\varepsilon^{3}\right) \\
b=\frac{35}{2} \pi\left(1+O\left(\varepsilon^{2}\right)\right)
\end{gathered}
$$

To compute the coefficient of the second order term in the expansion of $b$, we maintain one more term in the expansions of (25) and (26)

$$
\begin{gathered}
\text { RHS of }(25)=\varepsilon+\varepsilon^{3} \frac{143 \pi}{864}+O\left(\varepsilon^{4}\right), \quad k=3 \\
\text { RHS of }(26)=2 \pi\left(1+\frac{1}{4} \varepsilon^{2}+O\left(\varepsilon^{3}\right)\right)
\end{gathered}
$$

An equation for $b^{(2)}$ can be derived by substituting results (53) and (54) into Eq. (23) for $k=3$ :

$$
\frac{\varepsilon+(143 / 864) \varepsilon^{3}+O\left(\varepsilon^{4}\right)}{1+(1 / 4) \varepsilon^{2}+O\left(\varepsilon^{3}\right)}=\frac{\varepsilon}{1+b^{(2)} \varepsilon^{2}+O\left(\varepsilon^{3}\right)}
$$

which gives us $b^{(2)}=73 / 864$. So putting all these together, we find

$$
b=\frac{35}{2} \pi\left(1+\frac{73}{864} \varepsilon^{2}+O\left(\varepsilon^{4}\right)\right)
$$

To express the normalized Onsager potential $U(\theta)$ as a function of the normalized polymer concentration $b$, we solve for $\varepsilon$ from (55) and obtain

$$
\begin{gathered}
\varepsilon= \pm \sqrt{\frac{b-(35 / 2) \pi}{(2555 / 1728) \pi}} \\
U(\theta)=-\varepsilon \cos 6 \theta-\frac{35}{432} \varepsilon^{2} \cos 12 \theta+O\left(\varepsilon^{3}\right)
\end{gathered}
$$

The sign \pm indicates that there are two equivalent branches of nematic state near $b=(35 / 2) \pi$. These two branches are equivalent to each other via a 90 degree rotation. The equilibrium probability density is given by

$$
\rho(\theta)=\frac{1}{2 \pi}+\varepsilon \frac{35}{4 b} \cos 6 \theta+\varepsilon^{2} \frac{5005}{1728 b} \cos 12 \theta+O\left(\varepsilon^{3}\right)
$$

where $\varepsilon$ is given in (56). This is the third nematic branch for polymer ensembles with the Onsager potential. The behavior of this nematic branch is shown in Figure 5.

In Figure 5, the vertical coordinate $c_{6}$ gives a measure of the magnitude of the Onsager potential for the third branch while the equilibrium probability density is related to the Onsager potential by the Boltzmann distribution. Thus, $c_{6}$ also determines how far the nematic state deviates from the isotropic state. In this sense, $c_{6}$ can be treated as an unnormalized order parameter for the third branch. As we will see below, for the third branch, the order parameter $c_{6}$ actually measures the alignment of polymer rods with three directions. In Figure 5, the solid line denotes a very accurate numerical solution, which is treated as the exact solution, and the dashed line represents the asymptotic solution obtained above.

In Figure 6, we plot in polar coordinate system probability density $\rho(\theta)$ of a typical equilibrium state on the third

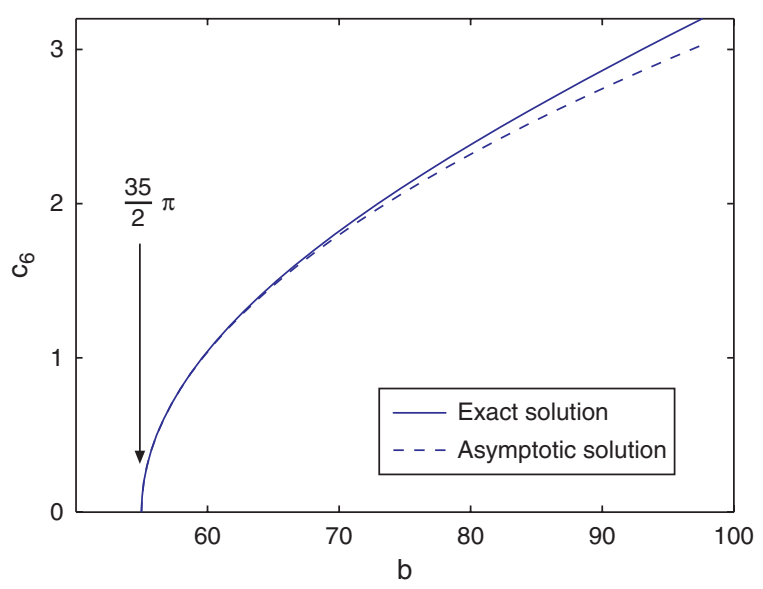

Fig. 5. The third nematic branch of polymer ensembles with the Onsager interaction potential. This nematic branch intersects with the isotropic branch at $(35 / 2) \pi$. The horizontal coordinate $b$ is the normalized polymer concentration; the vertical coordinate $c_{6}$ is the coefficient of the $\cos 6 \theta$ term (the leading non-zero term for the third nematic branch) in the Fourier expansion of the normalized Onsager potential. 


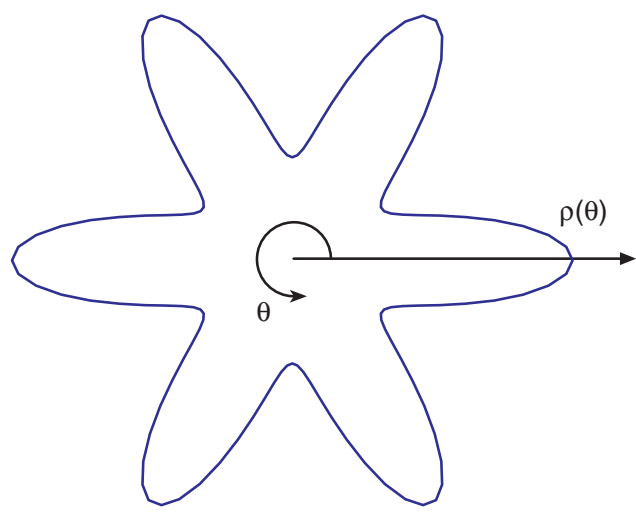

Fig. 6. Probability density $\rho(\theta)$ shown in polar coordinate system for a typical equilibrium state on the third nematic branch.

nematic branch. Function $f(\theta)$, the root-mean-square of the inner-product between polymer rods and the direction $\theta$, is not shown. For nematic branches other than the first nematic branch, we have $c_{2}=0$ and $f(\theta)=$ const. Figure 6 demonstrates that the probability density $\rho(\theta)$ has six fold symmetry and the polymer rods are equally likely being aligned along three directions that are uniformly distributed on the unit circle.

The process of finding nematic solutions can be continued indefinitely. Polymer rod ensemble with the Onsager intermolecular potential has infinitely many nematic branches. The first nematic branch intersects with the isotropic branch at $b=(3 / 2) \pi$, the second nematic branch at $b=(15 / 2) \pi$, the third nematic branch at $b=(35 / 2) \pi$. In general, if $\alpha_{2}=\alpha_{4}=\cdots=\alpha_{2(j-1)}=0$ but $\alpha_{2 j} \neq 0$, then in (29) for $k=j$ we must have

$$
1-\frac{\pi}{2 b^{(0)}}\left[(2 j)^{2}-1\right]=0
$$

which yields $b^{(0)}=\left(\left((2 j)^{2}-1\right) / 2\right) \pi$. Consequently, for $k>j$, we have $1-\pi /\left(2 b^{(0)}\right)\left[(2 k)^{2}-1\right]=$ $\left((2 j)^{2}-(2 k)^{2}\right) /\left((2 j)^{2}-1\right)<0$. It follows from (29) that $\alpha_{2 k}=0$ for $k>j$. Since $\alpha_{2 j} \neq 0$, we re-define $c_{2 j}=$ $\alpha_{2 j} \varepsilon+\beta_{2 j} \varepsilon^{2}+O\left(\varepsilon^{3}\right)$ as the new $\varepsilon$, which is equivalent to setting $\alpha_{2 j}=1$ and $\beta_{2 j}=0$. Substituting $\alpha_{2 j}=1$ and $\alpha_{2 k}=0$ for $k \neq j$ into (25), we have

RHS of (25)

$$
=\left(\varepsilon \alpha_{2 k}+\varepsilon^{2} \beta_{2 k}\right) \pi+\left\{\begin{array}{ll}
\varepsilon^{2} \frac{\pi}{4}, & k=2 j \\
0, & \text { otherwise }
\end{array}+O\left(\varepsilon^{3}\right)\right.
$$

To calculate coefficients $b^{(1)}$ and $\beta_{4 j}$, we keep both the first order terms and the second order terms in Eq. (23). For $k=j$, Eq. (23) becomes

$$
\frac{\varepsilon+O\left(\varepsilon^{3}\right)}{1+O\left(\varepsilon^{2}\right)}=\frac{\varepsilon+O\left(\varepsilon^{3}\right)}{1+\varepsilon b^{(1)}}
$$

which gives $b^{(1)}=0$. For $k=2 j$, (23) leads to an equation for $\beta_{4 j}$.

$$
\frac{\varepsilon^{2} \beta_{4 j}+\varepsilon^{2}(1 / 4)+O\left(\varepsilon^{3}\right)}{1+O\left(\varepsilon^{2}\right)}=\frac{(4 j)^{2}-1}{(2 j)^{2}-1} \cdot \frac{\varepsilon^{2} \beta_{4 j}}{1+O\left(\varepsilon^{2}\right)}
$$

which yields $\beta_{4 j}=(1 / 12) \cdot\left((2 j)^{2}-1\right) /\left((2 j)^{2}\right)$. For $k \notin$ $\{j, 2 j\}$, Eq. (23) has the form

$$
\frac{\varepsilon^{2} \beta_{2 k}+O\left(\varepsilon^{3}\right)}{1+O\left(\varepsilon^{2}\right)}=\frac{(2 k)^{2}-1}{(2 j)^{2}-1} \cdot \frac{\varepsilon^{2} \beta_{2 k}}{1+O\left(\varepsilon^{2}\right)}
$$

which, along with $\beta_{2 j}=0$, implies that $\beta_{2 k}=0$ for $k \neq 2 j$. Therefore, the nematic state that has zero coefficients for $\cos 2 \theta$ through $\cos 2(j-1) \theta$ but has a small non-zero coefficient for $\cos 2 j \theta$ in the Fourier expansion of $U(\theta)$ can be described mathematically by

$$
\begin{gathered}
U(\theta)=-\varepsilon \cos 2 j \theta-\frac{1}{12} \cdot \frac{(2 j)^{2}-1}{(2 j)^{2}} \varepsilon^{2} \cos 4 j \theta+O\left(\varepsilon^{3}\right) \\
b=\frac{(2 j)^{2}-1}{2} \pi\left(1+O\left(\varepsilon^{2}\right)\right)
\end{gathered}
$$

We would like to express the normalized Onsager potential $U(\theta)$ as a function of the normalized polymer concentration $b$. To achieve that goal, we need to express the small parameter $\varepsilon$ in terms of $b$. Since the first order term in the expansion of $b$ with respect to $\varepsilon$ has zero coefficient, to reveal the dependence of $b$ on $\varepsilon$, we need to find the leading term with non-zero coefficient. We re-visit the expansions of (25) and (26) and retain one more term in the expansions

$$
\text { RHS of }(25)=\varepsilon+\varepsilon^{3} \frac{\left(4(2 j)^{2}-1\right)}{24(2 j)^{2}} \pi+O\left(\varepsilon^{4}\right), \quad k=j
$$

$$
\text { RHS of }(26)=2 \pi\left(1+\frac{1}{4} \varepsilon^{2}+O\left(\varepsilon^{3}\right)\right)
$$

Substituting results (61) and (62) into Eq. (23) for $k=j$ gives us

$$
\frac{\varepsilon+\left(\left(4(2 j)^{2}-1\right)\right) /\left(24(2 j)^{2}\right) \varepsilon^{3}+O\left(\varepsilon^{4}\right)}{1+(1 / 4) \varepsilon^{2}+O\left(\varepsilon^{3}\right)}=\frac{\varepsilon}{1+b^{(2)} \varepsilon^{2}+O\left(\varepsilon^{3}\right)}
$$

Solving for $b^{(2)}$ from the equation above yields

$$
\begin{gathered}
b^{(2)}=\frac{2(2 j)^{2}+1}{24(2 j)^{2}} \\
b=\frac{(2 j)^{2}-1}{2} \pi\left(1+\frac{2(2 j)^{2}+1}{24(2 j)^{2}} \varepsilon^{2}+O\left(\varepsilon^{4}\right)\right)
\end{gathered}
$$

Now we use expansion (63) to express $\varepsilon$ in terms of the normalized polymer concentration $b$ and arrive at

$$
\begin{gathered}
\varepsilon= \pm \sqrt{\frac{b-\left(\left((2 j)^{2}-1\right) / 2\right) \pi}{\left(\left((2 j)^{2}-1\right)\left(2(2 j)^{2}+1\right)\right) /\left(48(2 j)^{2}\right) \pi}} \\
U(\theta)=-\varepsilon \cos 2 j \theta-\frac{1}{12} \cdot \frac{(2 j)^{2}-1}{(2 j)^{2}} \varepsilon^{2} \cos 4 j \theta+O\left(\varepsilon^{3}\right)
\end{gathered}
$$


Again, the sign \pm implies that there are two equivalent branches of nematic state near $b=\left(\left((2 j)^{2}-1\right) / 2\right) \pi$. These two branches are equivalent to each other via a 90 degree rotation. Once the Onsager potential is calculated, the equilibrium probability density is given by

$$
\begin{aligned}
\rho(\theta)= & \frac{1}{2 \pi}+\varepsilon \frac{(2 j)^{2}-1}{4 b} \cos 2 j \theta \\
& +\varepsilon^{2} \frac{\left((2 j)^{2}-1\right)\left(4(2 j)^{2}-1\right)}{48(2 j)^{2} b} \cos 4 j \theta+O\left(\varepsilon^{3}\right)
\end{aligned}
$$

where $\varepsilon$ is a function of the normalized polymer concentration $b$ given in (64). This is the $j$-th nematic branch of polymer ensembles with the Onsager interaction potential.

\section{NUMERICAL METHOD}

The asymptotic analysis in the previous section tells us two things: (i) there are infinitely many nematic branches for polymer ensembles with the Onsager interaction potential; and (ii) the $j$-th nematic branch intersects with the isotropic branch at $b=\left(\left((2 j)^{2}-1\right) / 2\right) \pi$. The approximate expression of each nematic branch near the intersection with the isotropic branch is obtained in the asymptotic analysis. In order to give a more accurate description of each nematic branch both near and away from the intersection with the isotropic branch, we need to solve (23) for an arbitrary value of $b$. In Eq. (23), $b$ is the parameter and the infinite sequence of coefficients $\left(c_{2}, c_{4}, \ldots, c_{2 j}, \ldots\right)$ is the unknown. From physical point of view, it is desirable to treat $\left(c_{2}, c_{4}, \ldots, c_{2 j}, \ldots\right)$ as functions of $b$. In the phase diagrams presented in the previous section, for practical reason, we show only the leading non-zero Fourier coefficient in $\left(c_{2}, c_{4}, \ldots, c_{2 j}, \ldots\right)$ as a function of $b$. Specifically, suppose

$$
c_{2}(b)=\cdots=c_{2(j-1)}(b)=0, \quad c_{2 j}(b)=r(b)
$$

In phase diagrams, we plot $r(b)$ as a function of $b$ and indicate that $r(b)$ is the coefficient for $\cos 2 j \theta$. Mathematically, however, it is difficult to solve for $r(b)$ in Eq. (23) for a given value of $b$. There are two mathematical difficulties: (i) as in the case of nematic polymers with the Maier-Saupe potential, $r(b)$ is not a single-valued function of $b$ and; (ii) function $r(b)$ is not defined at all for $b<(3 \pi / 2)$, that is, nematic state does not exist at all before the first nematic branch appears. Practically, for each nematic branch, it is more convenient to treat $r$ as the independent variable and treat $b$ as a function of $r$ instead. This convenient mathematical formulation has also been adopted for the study of nematic polymers with the MaierSaupe potential. ${ }^{3,45-47}$ For the case of the Maier-Saupe potential, the value of $r$ completely determines an equilibrium state. We believe that for the case of the Onsager potential, this is still true: the value of $r$ completely determines an equilibrium state on each given nematic branch.
This assertion has been confirmed in our numerical simulations. But a rigorous mathematical proof for the assertion is still open.

Now we present the numerical method we use to solve non-linear system (23). For the convenience of presentation, we introduce the vector notation

$$
\mathbf{C}=\left(c_{2}, c_{4}, \ldots, c_{2 k}, \ldots\right)
$$

Below we describe an iterative method for calculating the first nematic branch. Similar procedure can be employed to calculate other nematic branches. As we discussed above, in our new mathematical formulation, we treat $c_{2}=r$ as the independent variable and treat $b$ and $\left(c_{4}, \ldots, c_{2 k}, \ldots\right)$ as functions of $r$. In the numerical method, $r$ is the input, and $b$ and $\left(c_{4}, \ldots, c_{2 k}, \ldots\right)$ are the output. More precisely, we start with a given value of $r$ and proceed to calculate the corresponding values of $b(r)$ and $\left(c_{4}(r), \ldots, c_{2 k}(r), \ldots\right)$. Here is an outline of the iterative method:

- Step 0: We set $n=0$ and set $\mathbf{C}^{(0)}=(r, 0, \ldots, 0, \ldots)$. Here $n$ is the counter recording the number of iterations completed so far and $\mathbf{C}^{(0)}$ is the starting vector for iteration. - Step 1: We use $\mathbf{C}^{(n)}=\left(c_{2}^{(n)}, c_{4}^{(n)}, \ldots, c_{2 k}^{(n)}, \ldots\right)$ to calculate

$$
Q_{2 k}^{(n)}=\frac{\int_{0}^{2 \pi} \cos 2 k \theta \exp \left[\sum_{i=1}^{\infty} c_{2 i}^{(n)} \cos 2 i \theta\right] d \theta}{\int_{0}^{2 \pi} \exp \left[\sum_{i=1}^{\infty} c_{2 i}^{(n)} \cos 2 i \theta\right] d \theta}
$$

If $Q_{2 k}^{(n)}$ is the solution of Eq. (23), then for $k=1$, Eq. (23) becomes

$$
Q_{2}^{(n)}=\frac{3 \pi}{4 b} r
$$

Recall that in our mathematical formulation, $r$ (i.e., coefficient $c_{2}$ ) is the independent variable and $b$ is a function of $r$. We set the value of $b$ to satisfy this equation.

- Step 2: We calculate the new value of $b$

$$
b^{(n)}=\frac{3 \pi}{4 Q_{2}^{(n)}} r
$$

- Step 3: For $k>1$, we use Eq. (23) with the values of $Q_{2 k}^{(n)}$ and $b^{(n)}$ to update coefficient $c_{2 k}$ :

$$
c_{2 k}^{(n+1)}=\frac{4 Q_{2 k}}{\left(4 k^{2}-1\right) \pi} b^{(n)}, \quad \text { for } k>1
$$

- Step 4: We update the coefficient vector.

$$
\mathbf{C}^{(n+1)}=\left(r, c_{4}^{(n+1)}, \ldots, c_{2 k}^{(n+1)}, \ldots\right)
$$

- Step 5: We use $\left\|\mathbf{C}^{(n+1)}-\mathbf{C}^{(n)}\right\|$ to determine if the iteration has converged. If $\left\|\mathbf{C}^{(n+1)}-\mathbf{C}^{(n)}\right\|$ is less than the given tolerance, then we stop the iteration and output the most recent coefficient vector $\mathbf{C}^{(n+1)}$ and $b^{(n)}$ as the solution of Eq. (23). Otherwise, we increase the counter by one (set $n=n+1)$ and repeat the iteration starting at Step 1 . 


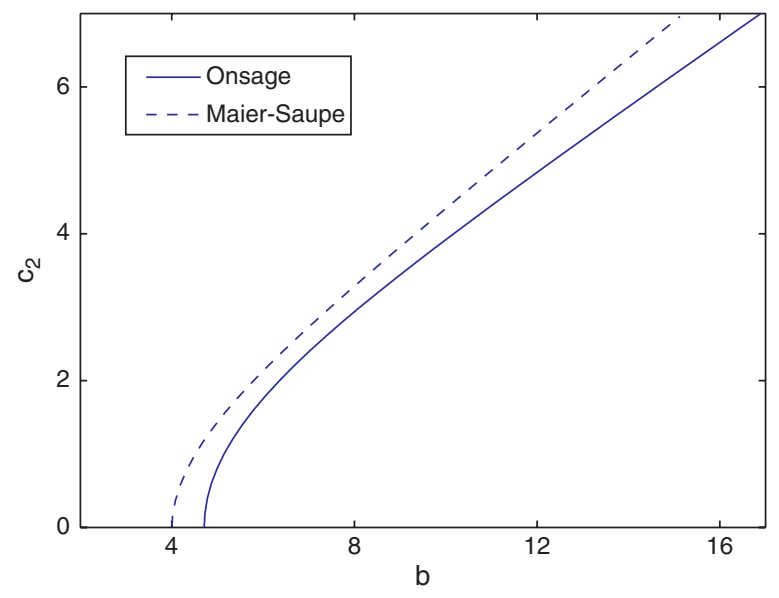

Fig. 7. Comparison of the first nematic branch for the Onsager potential and the only nematic branch for the Maier-Saupe potential.

Because the Smoluchowski equation is a diffusion equation we expect the probability density and the Onsager potential to be smooth functions. For a smooth function, the Fourier series expansion converges exponentially as the number of terms included in numerical solutions is increased. Therefore, numerical solutions accurate up to the computer precision can be obtained using the numerical method described above with only moderately large number of Fourier terms included. The numerical results are shown in Figures 1-6. Figure 7 compares the phase diagram for the Onsager potential (showing only the first nematic branch) and the phase diagram for the MaierSaupe potential. As shown in Figure 7, the Maier-Saupe potential predicts that the isotropic to nematic phase transition occurs at $b=4$ whereas the Onsager potential predicts that the isotropic to nematic phase transition occurs at $b=(3 / 2) \pi \approx 4.7124$.

\section{FREE ENERGY CALCULATION AND STABILITY OF THE ISOTROPIC BRANCH AND NEMATIC BRANCHES}

In the Doi-Hess model, the polymer orientation distribution is described by the probability density $\rho(\theta)$. The free energy of polymer orientation distribution $\rho(\theta)$ is

$$
\begin{aligned}
G([\rho]) & \equiv \frac{\text { Free energy of orientation distribution } \rho(\theta)}{k_{\mathrm{B}} T} \\
& =\int_{0}^{2 \pi} \rho(\theta)\left(\log \rho(\theta)+\frac{1}{2} U(\theta)\right) d \theta
\end{aligned}
$$

where $U(\theta)$ is the normalized Onsage interaction potential

$$
U(\theta)=b \int_{0}^{2 \pi}|\sin (\tilde{\theta}-\theta)| \rho(\tilde{\theta}) d \tilde{\theta}
$$

For the isotropic branch, the Onsager potential is $U(\theta)=0$, the probability density is $\rho(\theta)=1 /(2 \pi)$ and the corresponding free energy is

$$
G_{\text {Isotropic }}=-\log (2 \pi)
$$

Note that in the expression for the Onsager potential $U(\theta)=0$ we have dropped the constant term $4 b /(2 \pi)$. If we keep this constant term, then the free energy of the isotropic state is $G_{\text {Isotropic }}=(b / \pi)-\log (2 \pi)$, which increases linearly with the normalized polymer concentration $b$. Since the stability of an equilibrium state is determined by the free energy landscape at a fixed value of $b$, dropping a $b$-dependent constant term will not change the stability.

For an anisotropic orientation distribution (not necessarily an equilibrium state), the Fourier series expansion of probability density $\rho(\theta)$ and that of the normalized Onsager potential $U(\theta)$ are related by

$$
\begin{gathered}
\rho(\theta)=\frac{1}{2 \pi}+\sum_{j=1}^{\infty} a_{2 j} \cos 2 j \theta \\
U(\theta)=-\sum_{j=1}^{\infty} c_{2 j} \cos 2 j \theta \\
c_{2 j}=a_{2 j} \frac{4 b}{(2 j)^{2}-1}
\end{gathered}
$$

Here it is important to point out that relation (71) is true for all probability densities. In particular, the probability density $\rho(\theta)$ does not have to be an equilibrium state. If $\rho(\theta)$ is an equilibrium probability density, then in addition to relation (71), we also have the Boltzmann relation

$$
\log \rho(\theta)=-U(\theta)-\log (Z)=\sum_{j=1}^{\infty} c_{2 j} \cos 2 j \theta-\log (Z)
$$

where the partition function $Z$ is given by

$$
Z=\int_{0}^{2 \pi} \exp \left(\sum_{j=1}^{\infty} c_{2 j} \cos 2 j \theta\right) d \theta
$$

The free energy of an equilibrium state is

$$
\begin{aligned}
G([\rho]) & =\int_{0}^{2 \pi} \rho(\theta)\left(\frac{1}{2} \sum_{j=1}^{\infty} c_{2 j} \cos 2 j \theta-\log (Z)\right) d \theta \\
& =\frac{\pi}{2} \sum_{j=1}^{\infty} c_{2 j} a_{2 j}-\log (Z) \\
& =\frac{\pi}{8 b} \sum_{j=1}^{\infty} c_{2 j}^{2}\left((2 j)^{2}-1\right)-\log (Z)
\end{aligned}
$$

For the first nematic branch, the Onsager potential has the form $U(\theta)=-\left(\varepsilon \cos 2 \theta+(1 / 16) \varepsilon^{2} \cos 4 \theta+\cdots\right)$ and the partition function is

$$
\begin{gathered}
Z=\int_{0}^{2 \pi} \exp \left(\varepsilon \cos 2 \theta+\frac{1}{16} \varepsilon^{2} \cos 4 \theta+\cdots\right) d \theta \\
=\int_{0}^{2 \pi}\left(1+\frac{1}{2} \varepsilon^{2} \cos ^{2} 2 \theta+\frac{1}{2} \cdot \frac{1}{16^{2}} \varepsilon^{4} \cos ^{2} 4 \theta\right. \\
+\frac{1}{6} \cdot \frac{3}{16} \varepsilon^{4} \cos ^{2} 2 \theta \cos 4 \theta
\end{gathered}
$$

J. Comput. Theor. Nanosci. 7, 1-18, 2010 


$$
\begin{array}{r}
\left.+\frac{1}{24} \varepsilon^{4} \cos ^{4} 2 \theta+\cdots\right) d \theta \\
=2 \pi\left(1+\frac{1}{4} \varepsilon^{2}+\frac{25}{1024} \varepsilon^{4}+\cdots\right)
\end{array}
$$

The log partition function takes the form

$$
\begin{aligned}
\log (Z) & =\log (2 \pi)+\log \left(1+\frac{1}{4} \varepsilon^{2}+\frac{25}{1024} \varepsilon^{4}+\cdots\right) \\
& =\log (2 \pi)+\left(\frac{1}{4} \varepsilon^{2}+\frac{25}{1024} \varepsilon^{4}\right)-\frac{1}{2}\left(\frac{1}{4} \varepsilon^{2}\right)^{2}+\cdots \\
& =\log (2 \pi)+\left(\frac{1}{4} \varepsilon^{2}-\frac{7}{1024} \varepsilon^{4}\right)+\cdots
\end{aligned}
$$

The free energy of an equilibrium state on the first nematic branch is

$$
\begin{aligned}
G_{\text {First }}= & \frac{\pi}{8 b}\left(3 \varepsilon^{2}+\frac{15}{256} \varepsilon^{4}\right)-\left(\frac{1}{4} \varepsilon^{2}-\frac{7}{1024} \varepsilon^{4}\right) \\
& -\log (2 \pi)+\cdots \\
= & \frac{3 \pi}{8 b}\left[\varepsilon^{2}+\frac{5}{256} \varepsilon^{4}-\left(\varepsilon^{2}-\frac{7}{256} \varepsilon^{4}\right) \frac{2 b}{3 \pi}+\cdots\right] \\
& \quad-\log (2 \pi)
\end{aligned}
$$

Using the fact that for the first nematic branch $b=(3 \pi) /$ $2\left(1+(3 / 32) \varepsilon^{2}\right)$, we obtain

$$
\begin{aligned}
G_{\text {First }}= & \frac{3 \pi}{8 b}\left[\varepsilon^{2}+\frac{5}{256} \varepsilon^{4}-\left(\varepsilon^{2}+\frac{17}{256} \varepsilon^{4}\right)+\cdots\right]-\log (2 \pi) \\
= & -\frac{3 \pi}{8 b} \cdot \frac{12}{256} \varepsilon^{4}-\log (2 \pi)+\cdots \\
= & \frac{9 \pi}{512} \cdot \frac{1}{b} \varepsilon^{4}-\log (2 \pi)+\cdots \\
= & -\frac{9 \pi}{512} \cdot \frac{1}{b}\left(\frac{b-(3 / 2) \pi}{(9 / 64) \pi}\right)^{2}-\log (2 \pi) \\
& \quad+\cdots<G_{\text {Isotropic }}
\end{aligned}
$$

Since $G_{\text {Isotropic }}+\log (2 \pi)=0$, for simplicity of presentation, we plot $G+\log (2 \pi)$ as the free energy. Figure 8 shows the free energy of the first nematic branch as a function of the normalized polymer concentration $b$. The solid line represents a very accurate numerical solution obtained using the numerical method described in the previous section, which is treated as the exact solution, and the dashed line represents the asymptotic solution obtained above. It is clear that the asymptotic solution is a very good approximation to the exact solution over a very large range of $b$. Actually, the difference between the asymptotic solution and the exact solution will not increase when $b$ is increased further (see Fig. 9).

In Figure 8, the dotted line shows the free energy of the isotropic branch. Notice that the free energy of the first nematic branch is below the free energy of the isotropic

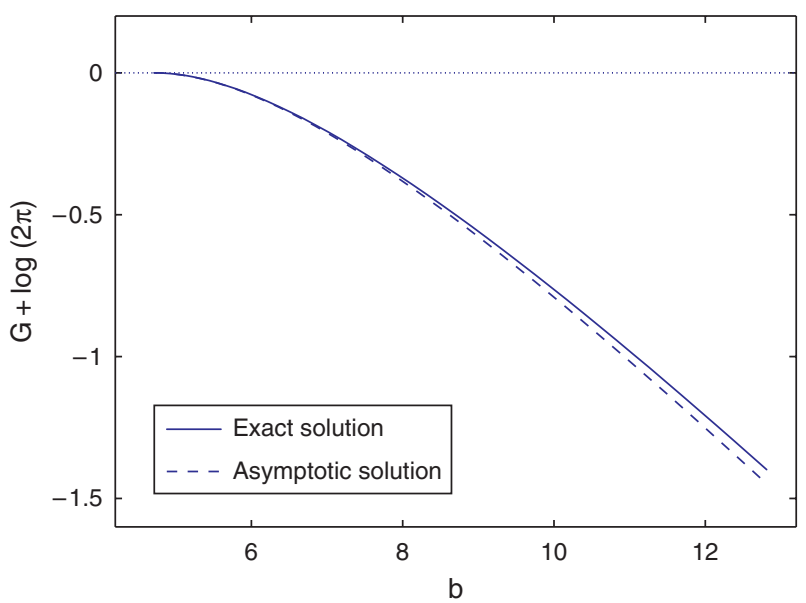

Fig. 8. Free energy of equilibrium state on the first nematic branch as a function of the normalized polymer concentration $b$.

branch. The first nematic branch appears at $b=(3 / 2) \pi$. For $b>(3 / 2) \pi$ but close to $(3 / 2) \pi$, the first nematic branch is the only nematic branch. Therefore, the lower free ehergy of the first nematic branch implies stability. For $b<(3 / 2) \pi$, the isotropic branch is the only solution. As a result, for $b<(3 / 2) \pi$, the isotropic branch is stable. The stability of the isotropic branch for $b>(3 / 2) \pi$ will be analyzed later in this section.

For the $j$-th nematic branch, the Onsager potential has the form $U(\theta)=-\left(\varepsilon \cos 2 j \theta+\left((2 j)^{2}-1\right) /\right.$ $\left.\left(12(2 j)^{2}\right) \varepsilon^{2} \cos 4 j \theta+\cdots\right)$ and the partition function is

$$
\begin{gathered}
Z=\int_{0}^{2 \pi} \exp \left(\varepsilon \cos 2 j \theta+\frac{(2 j)^{2}-1}{12(2 j)^{2}} \varepsilon^{2} \cos 4 j \theta+\cdots\right) d \theta \\
=\int_{0}^{2 \pi}\left(1+\frac{1}{2} \varepsilon^{2} \cos ^{2} 2 j \theta+\frac{1}{2} \cdot \frac{\left((2 j)^{2}-1\right)^{2}}{12^{2}(2 j)^{4}} \varepsilon^{4} \cos ^{2} 4 j \theta\right. \\
+\frac{3}{6} \cdot \frac{(2 j)^{2}-1}{12(2 j)^{2}} \varepsilon^{4} \cos ^{2} 2 j \theta \cos 4 j \theta \\
\left.\quad+\frac{1}{24} \varepsilon^{4} \cos ^{4} 2 j \theta+\cdots\right) d \theta \\
=2 \pi\left(1+\frac{1}{4} \varepsilon^{2}+\frac{\left(4(2 j)^{2}-1\right)^{2}}{9 \cdot 64(2 j)^{4}} \varepsilon^{4}+\cdots\right)
\end{gathered}
$$

The log partition function has the expression

$$
\begin{aligned}
\log (Z)= & \log (2 \pi)+\log \left(1+\frac{1}{4} \varepsilon^{2}+\frac{\left(4(2 j)^{2}-1\right)^{2}}{9 \cdot 64(2 j)^{4}} \varepsilon^{4}+\cdots\right) \\
= & \log (2 \pi)+\left(\frac{1}{4} \varepsilon^{2}+\frac{\left(4(2 j)^{2}-1\right)^{2}}{9 \cdot 64(2 j)^{4}} \varepsilon^{4}\right) \\
& \quad-\frac{1}{2}\left(\frac{1}{4} \varepsilon^{2}\right)^{2}+\cdots \\
= & \log (2 \pi)+\left(\frac{1}{4} \varepsilon^{2}-\frac{2\left((2 j)^{2}+2\right)^{2}-9}{9 \cdot 64(2 j)^{4}} \varepsilon^{4}\right)+\cdots
\end{aligned}
$$




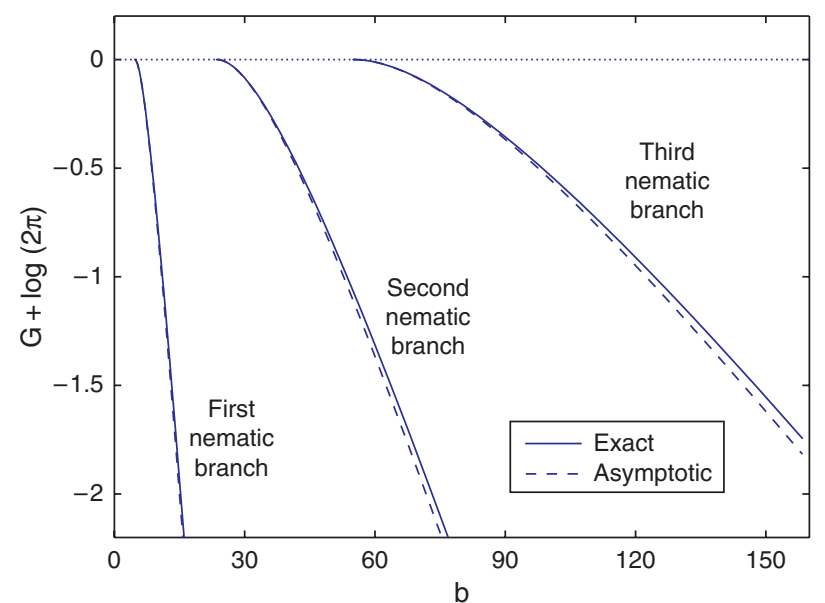

Fig. 9. Free energies of the first three nematic branches as functions of the normalized polymer concentration $b$. The dotted line is the free energy of the isotropic branch.

The free energy of an equilibrium state on the first nematic branch is given by

$$
\begin{aligned}
G_{j \text {-h }}=\frac{\pi}{8 b}\left(\left((2 j)^{2}-1\right) \varepsilon^{2}+\frac{\left(4(2 j)^{2}-1\right)\left((2 j)^{2}-1\right)^{2}}{9 \cdot 16(2 j)^{4}} \varepsilon^{4}\right) \\
-\left(\frac{1}{4} \varepsilon^{2}-\frac{2\left((2 j)^{2}+2\right)^{2}-9}{9 \cdot 64(2 j)^{4}} \varepsilon^{4}\right)-\log (2 \pi)+\cdots \\
=\frac{\left((2 j)^{2}-1\right) \pi}{8 b}\left[\varepsilon^{2}+\frac{4(2 j)^{4}-5(2 j)^{2}+1}{9 \cdot 16(2 j)^{4}} \varepsilon^{4}\right. \\
-\left(\varepsilon^{2}-\frac{2\left((2 j)^{2}+2\right)^{2}-9}{9 \cdot 16(2 j)^{4}} \varepsilon^{4}\right) \\
\left.\times \frac{2 b}{\left((2 j)^{2}-1\right) \pi}+\cdots\right]-\log (2 \pi)
\end{aligned}
$$

Using the fact that for the $j$-th nematic branch $b=\left((2 j)^{2}-1\right) / 2 \pi\left(1+\left(2(2 j)^{2}+1\right) /\left(24(2 j)^{2}\right) \varepsilon^{2}+\right.$ $\left.O\left(\varepsilon^{4}\right)\right)$, we obtain

$$
\begin{aligned}
G_{j-\mathrm{th}}= & \frac{\left((2 j)^{2}-1\right) \pi}{8 b}\left[\varepsilon^{2}+\frac{4(2 j)^{4}-5(2 j)^{2}+1}{9 \cdot 16(2 j)^{4}} \varepsilon^{4}\right. \\
& -\left(\varepsilon^{2}-\frac{2\left((2 j)^{2}+2\right)^{2}-9}{9 \cdot 16(2 j)^{4}} \varepsilon^{4}\right) \\
& \left.\times \frac{2 b}{\left((2 j)^{2}-1\right) \pi}+\cdots\right]-\log (2 \pi) \\
= & -\frac{\left((2 j)^{2}-1\right) \pi}{8 b} \cdot \frac{6(2 j)^{2}+3}{9 \cdot 16(2 j)^{2}} \varepsilon^{4}-\log (2 \pi)+\cdots \\
= & -\frac{2(2 j)^{4}-(2 j)^{2}-1}{3 \cdot 128(2 j)^{2}} \pi \cdot \frac{1}{b} \varepsilon^{4}-\log (2 \pi)+\cdots \\
= & -\frac{2(2 j)^{4}-(2 j)^{2}-1}{384(2 j)^{2}} \pi
\end{aligned}
$$

$$
\begin{aligned}
& \cdot \frac{1}{b}\left(\frac{b-\left(\left((2 j)^{2}-1\right) / 2\right) \pi}{\left(\left((2 j)^{2}-1\right)\left(2(2 j)^{2}+1\right)\right) /\left(48(2 j)^{2}\right) \pi}\right)^{2} \\
&-\log (2 \pi)+\cdots \\
&<G_{\text {Isotropic }}
\end{aligned}
$$

Figure 9 shows the free energy of the first three nematic branches. It is clear that for all nematic branches, the free energy is below that of the isotropic branch, which was established analytically in (82) above. The first nematic branch has the lowest free energy. The higher the index the higher the free energy. The free energy of the second nematic branch is above that of the first branch and the free energy of the third nematic branch is above that of the second branch and so on.

Figure 9 also demonstrates that the asymptotic expression (82) is valid for a very large range of $b$ and valid for all nematic branches. The asymptotic solution is derived based on the assumption that the amplitude of the Onsager potential is small. So it is still a mystery why the difference between the asymptotic solution and the exact solution remains small as the amplitude of the Onsager potential is increased over a very large range corresponding to the increase in $b$. The mathematical explanation behind this mystery will be investigated in a future study.

We use free energy calculation to study the stability of the isotropic branch for $b>(3 / 2) \pi$ (i.e., after the first nematic branch appears). The free energy diagram (Fig. 9) suggests that the isotropic state is unstable for $b>(3 / 2) \pi$. To show the instability, it is sufficient to show that it is unstable with respect to a perturbation of one particular form. We consider a perturbation to the isotropic state of the form

$$
\rho(\theta)=\frac{1}{2 \pi}(1+\eta \cos 2 \theta)
$$

where $\eta$ is a small parameter. The perturbed probability density satisfies $\int_{0}^{\infty} \rho(\theta) d \theta=1$. Since in general the perturbed probability density is no longer an equilibrium state, we cannot apply the Boltzmann relation. To calculate the free energy of the perturbed probability density, we cannot use the expression given in (74) for equilibrium states. Instead we have to go back to the free energy expression given in (68) for a general polymer orientation distribution (not necessarily an equilibrium state). Recall that the Fourier series expansion of $\rho(\theta)$ and the Fourier series expansion of $U(\theta)$ are connected by (71). The Onsager potential corresponding to the perturbed probability density is

$$
U(\theta)=-\left(\frac{\eta}{2 \pi}\right) \cdot \frac{4 b}{3} \cos 2 \theta=-\eta \frac{2 b}{3 \pi} \cos 2 \theta
$$

The second component (interaction potential) of the free energy expression given in (68) is 


$$
\begin{aligned}
\int_{0}^{\infty} \rho(\theta)\left(\frac{1}{2} U(\theta)\right) d \theta & =\int_{0}^{\infty} \rho(\theta)\left(-\eta \frac{b}{3 \pi} \cos 2 \theta\right) d \theta \\
& =-\frac{\eta}{2 \pi} \cdot \eta \frac{b}{3 \pi} \cdot \pi=\eta^{2} \frac{b}{6 \pi}
\end{aligned}
$$

The first component (entropic contribution) of the free energy expression given in (68) is

$$
\begin{aligned}
& \int_{0}^{2 \pi} \rho(\theta) \log \rho(\theta) d \theta \\
& =\int_{0}^{2 \pi} \rho(\theta) \log (1+\eta \cos 2 \theta) d \theta-\log (2 \pi) \\
& =\int_{0}^{2 \pi} \rho(\theta)\left(\eta \cos 2 \theta-\frac{\eta^{2}}{2} \cos ^{2} 2 \theta+O\left(\eta^{3}\right)\right) d \theta+G_{\text {isotropic }} \\
& =\frac{\eta}{2 \pi} \cdot \eta \cdot \pi-\frac{\eta^{2}}{2} \cdot \frac{1}{2}-\log (2 \pi)+O\left(\eta^{3}\right) \\
& =\frac{\eta^{2}}{4}+O\left(\eta^{3}\right)+G_{\text {isotropic }}
\end{aligned}
$$

The total free energy of the perturbed probability density is

$$
G(\eta)=G_{\text {isotropic }}-\frac{\eta^{2}}{6 \pi}\left(b-\frac{3 \pi}{2}\right)+O\left(\eta^{3}\right)
$$

For fixed $b>(3 \pi) / 2$ and small $\eta$, we have $G(\eta)<$ $G_{\text {isotropic }}$, which implies that for $b>(3 \pi) / 2$ the isotropic state is unstable.

So far we have concluded that (i) the isotropic state is the only solution for $b<(3 \pi) / 2$ and is stable for $b<(3 \pi) / 2$; (ii) the first nematic branch appears at $b=(3 \pi) / 2$ and is stable for $b>(3 \pi) / 2$. The $j$-th nematic branch appears at $b=\left(\left((2 j)^{2}-1\right) / 2\right) \pi$. Although the free energy diagram (Fig. 9) above does not yield a definite conclusion on the stability of the $j$-th nematic branch for $j>1$, intuitively it is reasonable to expect that for $j>1$ the $j$-th nematic branch is unstable. Below we use free energy calculation to show the instability. Again, to conclude the instability, we only need to identify one unstable model. For $j>1$, the unperturbed equilibrium probability density on the $j$-th nematic branch has the form

$$
\rho^{(e)}(\theta)=\frac{1}{2 \pi}+\sum_{k=2}^{\infty} a_{2 k} \cos 2 k \theta
$$

Specifically, the unperturbed probability density satisfies $a_{2}=0$. We consider a perturbation to the equilibrium probability density of the form

$$
\rho(\theta)=\rho^{(e)}(\theta)+\eta \cos 2 \theta
$$

where $\eta$ is a small parameter. The perturbed probability density satisfies $\int_{0}^{2 \pi} \rho(\theta) d \theta=1$. To proceed with the free energy calculation, we first look at the expansion of $x \cdot \log x$

$(x+\delta) \cdot \log (x+\delta)=x \cdot \log x+(\log x+1) \delta+\frac{1}{2} \cdot \frac{1}{x} \delta^{2}+\cdots$
We expand the free energy as a power series of $\eta$ and keep only the constant terms and $\eta^{2}$ terms. Note that all $\eta$ terms sum to zero because the unperturbed probability density is an equilibrium. The expansion of the first component (entropic contribution) of free energy is

$$
\begin{aligned}
\int_{0}^{2 \pi}\left(\rho^{(e)}(\theta)+\eta \cos 2 \theta\right) \log \left(\rho^{(e)}(\theta)+\eta \cos 2 \theta\right) d \theta \\
=\int_{0}^{2 \pi}\left(\rho^{(e)}(\theta) \log \rho^{(e)}(\theta)\right. \\
\left.\quad+\frac{1}{2} \frac{1}{\rho^{(e)}(\theta)}(\eta \cos 2 \theta)^{2}+O\left(\eta^{3}\right)\right) d \theta \\
=\int_{0}^{2 \pi} \rho^{(e)}(\theta) \log \rho^{(e)}(\theta) d \theta \\
\quad+\frac{1}{4} \eta^{2} \int_{0}^{2 \pi} \frac{1}{\rho^{(e)}(\theta)}(1+\cos 4 \theta) d \theta+O\left(\eta^{3}\right)
\end{aligned}
$$

The Onsager potential corresponding to the perturbed probability density is

$$
U(\theta)=U^{(e)}(\theta)-\eta \cdot \frac{4 b}{3} \cos 2 \theta
$$

where $U^{(e)}(\theta)$ is the Onsager potential corresponding to the unperturbed equilibrium probability density given by

$$
U^{(e)}(\theta)=-\sum_{k=2}^{\infty} a_{2 k} \frac{4 b}{(2 k)^{2}-1} \cos 2 k \theta
$$

We expand the second component (the Onsager potential) of the free energy.

$$
\begin{gathered}
\frac{1}{2} \int_{0}^{2 \pi}\left(\rho^{(e)}(\theta)+\eta \cos 2 \theta\right)\left(U^{(e)}(\theta)-\eta \cdot \frac{4 b}{3} \cos 2 \theta\right) d \theta \\
=\frac{1}{2} \int_{0}^{2 \pi}\left(\rho^{(e)}(\theta) U^{(e)}(\theta) d \theta-\frac{4 b}{3} \eta^{2} \cos ^{2} 2 \theta\right) d \theta \\
=\frac{1}{2} \int_{0}^{2 \pi} \rho^{(e)}(\theta) U^{(e)}(\theta) d \theta-\frac{2 \pi b}{3} \eta^{2}
\end{gathered}
$$

Adding together the two components of the free energy, we have

$$
\begin{aligned}
G(\eta)= & G(0)-\eta^{2} \pi^{2} \\
& \times\left(\frac{2 b}{3 \pi}-\frac{1}{4 \pi^{2}} \int_{0}^{2 \pi} \frac{1}{\rho^{(e)}(\theta)}(1+\cos 4 \theta) d \theta\right)+O\left(\eta^{3}\right)
\end{aligned}
$$

For the $j$-th nematic branch, the equilibrium probability density is

$$
\rho^{(e)}=\frac{1}{2 \pi}\left(1+\varepsilon q_{2 j} \cos 2 j \theta+\varepsilon^{2} r_{2 j} \cos 4 j \theta+O\left(\varepsilon^{3}\right)\right)
$$


where $\varepsilon$ and coefficients $q_{2 j}$ and $r_{2 j}$ are given by

$$
\begin{gathered}
\varepsilon= \pm \sqrt{\frac{b-\left(\left((2 j)^{2}-1\right) / 2\right) \pi}{\left(\left((2 j)^{2}-1\right)\left(2(2 j)^{2}+1\right)\right) /\left(48(2 j)^{2}\right) \pi}} \\
q_{2 j}=2 \pi \frac{(2 j)^{2}-1}{4 b} \\
r_{2 j}=2 \pi \frac{\left((2 j)^{2}-1\right)\left(4(2 j)^{2}-1\right)}{48(2 j)^{2} b}
\end{gathered}
$$

The reciprocal of the equilibrium probability density has the expansion.

$$
\begin{gathered}
\frac{1}{\rho^{(e)}(\theta)}=2 \pi\left(1-\varepsilon q_{2 j} \cos 2 j \theta-\varepsilon^{2} r_{2 j} \cos 4 j \theta\right. \\
\left.+\varepsilon^{2} q_{2 j}^{2} \cos ^{2} 2 j \theta+O\left(\varepsilon^{3}\right)\right)
\end{gathered}
$$

We calculate the second term in the coefficient of $\eta^{2}$ in (94).

$$
\begin{aligned}
\frac{1}{4 \pi^{2}} \int_{0}^{2 \pi} \frac{1}{\rho^{(e)}(\theta)}(1+\cos 4 \theta) d \theta & \\
& = \begin{cases}1+\varepsilon^{2}\left(\frac{3}{4} q_{2}^{2}-\frac{1}{2} r_{2}\right)+\cdots & \text { for } j=1 \\
1-\varepsilon \frac{1}{2} q_{4}+\varepsilon^{2} \frac{1}{2} q_{4}^{2}+\cdots & \text { for } j=2 \\
1+\varepsilon^{2} \frac{1}{2} q_{2 j}^{2}+\cdots & \text { for } j>2\end{cases}
\end{aligned}
$$

The $j$-th nematic exists only for $b>\left(\left((2 j)^{2}-1\right) / 2\right) \pi$. Thus, for $j>1$ and for $b$ near $\left(\left((2 j)^{2}-1\right) / 2\right) \pi$ (i.e., for nematic states of small amplitude $\varepsilon$ ), we have

$$
\begin{aligned}
G(\eta) & <G(0)-\eta^{2} \pi^{2}\left(\frac{(2 j)^{2}-1}{3}-1+O(\varepsilon)\right)+O\left(\eta^{3}\right) \\
& <G(0)-2 \eta^{2} \pi^{2}+O\left(\eta^{3}\right)<G(0)
\end{aligned}
$$

which implies that for $j>1$, the $j$-th nematic branch is unstable. In the above, we have concluded the stability of the first nematic branch based on the fact that it is the equilibrium state with the lowest free energy. Nevertheless, it is worthwhile to examine directly the stability of the first nematic branch in the presence of a small perturbation. We consider perturbed probability densities of the form

$$
\rho(\theta)=\rho^{(e)}(\theta)+\eta \sum_{k=1}^{\infty} \lambda_{2 k} \cos 2 k \theta
$$

It can be shown that including Fourier modes $\cos (2 k+1) \theta$ and/or $\sin k \theta$ in the perturbation will only increase the free energy. Consequently, to derive the stability we only need to consider perturbations consisting of Fourier modes $\cos 2 k \theta$. The Onsager potential corresponding to the perturbed probability density is

$$
U(\theta)=U^{(e)}(\theta)-\eta \sum_{k=1}^{\infty} \frac{4 b}{(2 k)^{2}-1} \lambda_{2 k} \cos 2 k \theta
$$

where $U^{(e)}(\theta)$ is the Onsager potential corresponding to the unperturbed probability density. We expand the two components of the free energy as a power series of $\eta$. Again, we collect only the constant terms and $\eta^{2}$ terms. The sum of all $\eta$ terms must be zero because the unperturbed probability density is an equilibrium state.

$$
\begin{aligned}
& G(\eta)=\int_{0}^{2 \pi} \rho(\theta)\left(\frac{1}{2} U(\theta)+\log \rho(\theta)\right) d \theta \\
& =\int_{0}^{2 \pi}\left[\frac{1}{2} \rho^{(e)}(\theta) U^{(e)}(\theta)-2 b \eta^{2}\left(\sum_{k=1}^{\infty} \lambda_{2 k} \cos 2 k \theta\right)\right. \\
& \times\left(\sum_{k=1}^{\infty} \frac{\lambda_{2 k} \cos 2 k \theta}{(2 k)^{2}-1}\right)+\rho^{(e)}(\theta) \log \rho^{(e)}(\theta) \\
& \left.+\frac{1}{2} \frac{1}{\rho^{(e)}(\theta)} \eta^{2}\left(\sum_{k=1}^{\infty} \lambda_{2 k} \cos 2 k \theta\right)^{2}\right] d \theta+O\left(\eta^{3}\right) \\
& =G(0)-2 \pi b \eta^{2} \sum_{k=2}^{\infty} \frac{\lambda_{2 k}^{2}}{(2 k)^{2}-1} \\
& +\pi^{2} \eta^{2} \lambda_{2}^{2}\left(\frac{1}{2 \pi^{2}} \int_{0}^{2 \pi} \frac{\cos ^{2} 2 \theta}{\rho^{(e)}(\theta)} d \theta-\frac{2 b}{3 \pi}\right) \\
& +\eta^{2} \int_{0}^{2 \pi} \frac{\lambda_{2} \cos 2 \theta}{\rho^{(e)}(\theta)}\left(\sum_{k=2}^{\infty} \lambda_{2 k} \cos 2 k \theta\right) d \theta \\
& +\frac{1}{2} \eta^{2} \int_{0}^{2 \pi} \frac{1}{\rho^{(e)}(\theta)}\left(\sum_{k=2}^{\infty} \lambda_{2 k} \cos 2 k \theta\right)^{2} d \theta+O\left(\eta^{3}\right) \\
& \equiv G(0)+\pi^{2} \eta^{2} T_{1}+\pi^{2} \eta^{2} T_{2}+\pi^{2} \eta^{2} T_{3} \\
& +\pi^{2} \eta^{2} T_{4}+O\left(\eta^{3}\right)
\end{aligned}
$$

We calculate $T_{1}, T_{2}, T_{3}$, and $T_{4}$ one by one. $T_{1}$ is bounded by $T_{1}=-\frac{2 b}{\pi} \sum_{k=2}^{\infty} \frac{\lambda_{2 k}^{2}}{(2 k)^{2}-1} \geq-\frac{2 b}{\pi} \sum_{k=2}^{\infty} \frac{\lambda_{2 k}^{2}}{15}=-\frac{2 b}{15 \pi} \sum_{k=2}^{\infty} \lambda_{2 k}^{2}$

For $b$ near $(3 \pi) / 2$ (i.e., for nematic states of small amplitude $\varepsilon$ on the first branch), we have

$$
T_{1} \approx-\frac{1}{5} \sum_{k=2}^{\infty} \lambda_{2 k}^{2} \geq-\frac{2}{7} \sum_{k=2}^{\infty} \lambda_{2 k}^{2}
$$

For $b$ near $(3 \pi) / 2$, we use $(b-(3 \pi) / 2)=\varepsilon^{2}(9 \pi) / 64$ and result (98) to expand $T_{2}$ with respect to $\varepsilon$

$$
\begin{aligned}
T_{2} & =\lambda_{2}^{2}\left(\frac{1}{2 \pi^{2}} \int_{0}^{2 \pi} \frac{\cos ^{2} 2 \theta}{\rho^{(e)}(\theta)} d \theta-\frac{2 b}{3 \pi}\right) \\
& \approx \lambda_{2}^{2} \varepsilon^{2}\left[\left(\frac{3}{4} q_{2}^{2}-\frac{1}{2} r_{2}\right)-\frac{3}{32}\right]
\end{aligned}
$$

At $b=(3 \pi) / 2$ where the first nematic branch emerges from the isotropic branch, coefficients $q_{2}$ and $r_{2}$ take the values $q_{2}=1$ and $r_{2}=5 / 16$, which leads to

$$
\left.\left[\left(\frac{3}{4} q_{2}^{2}-\frac{1}{2} r_{2}\right)-\frac{3}{32}\right]\right|_{b=(3 \pi) / 2}=\frac{1}{2}>\frac{3}{7}
$$

J. Comput. Theor. Nanosci. 7, 1-18, 2010 
It follows that for $b$ near $(3 \pi) / 2, T_{2}$ has a lower bound given by

$$
T_{2} \geq \frac{3}{7} \varepsilon^{2} \lambda_{2}^{2}
$$

For $b$ near $(3 \pi) / 2$, the nematic equilibrium probability density on the first branch has the expansion given in (95). Using that expansion, we derive lower bounds for $T_{3}$ and $T_{4}$.

$$
\begin{aligned}
T_{3} & =\frac{1}{\pi^{2}} \int_{0}^{2 \pi} \frac{\lambda_{2} \cos 2 \theta}{\rho^{(e)}(\theta)}\left(\sum_{k=2}^{\infty} \lambda_{2 k} \cos 2 k \theta\right) d \theta \\
& =-\frac{2}{\pi} \varepsilon q_{2} \lambda_{2} \lambda_{4} \int_{0}^{2 \pi} \cos ^{2} 2 \theta \cos 4 \theta d \theta+O\left(\varepsilon^{2}\right) \\
& \approx-\varepsilon \lambda_{2} \lambda_{4}>-\frac{4}{7} \sqrt{\varepsilon^{2} \lambda_{2}^{2}} \cdot \sqrt{\sum_{k=2}^{\infty} \lambda_{2 k}^{2}} \\
T_{4} & =\frac{1}{\pi^{2}} \frac{1}{2} \int_{0}^{2 \pi} \frac{1}{\rho^{(e)}(\theta)}\left(\sum_{k=2}^{\infty} \lambda_{2 k} \cos 2 k \theta\right)^{2} d \theta \\
& =\frac{1}{\pi} \int_{0}^{2 \pi}\left(\sum_{k=2}^{\infty} \lambda_{2 k} \cos 2 k \theta\right)^{2} d \theta+O(\varepsilon) \\
& \approx \sum_{k=2}^{\infty} \lambda_{2 k}^{2} \geq \frac{6}{7} \sum_{k=2}^{\infty} \lambda_{2 k}^{2}
\end{aligned}
$$

Substituting all these results into (102), we arrive at

$$
\begin{aligned}
G(\eta) \geq & G(0)+\eta^{2} \frac{\pi^{2}}{7} \\
& \times\left(4 \sum_{k=2}^{\infty} \lambda_{2 k}^{2}-4 \sqrt{\varepsilon^{2} \lambda_{2}^{2}} \cdot \sqrt{\sum_{k=2}^{\infty} \lambda_{2 k}^{2}}+3 \varepsilon^{2} \lambda_{2}^{2}\right) \\
\geq & G(0)+\eta^{2} \frac{\pi^{2}}{7}\left(2 \sum_{k=2}^{\infty} \lambda_{2 k}^{2}+\varepsilon^{2} \lambda_{2}^{2}\right)>G(0)
\end{aligned}
$$

which concludes the stability of the first nematic branch.

\section{CONCLUSIONS}

We have carried out an asymptotic analysis on the phase diagram of nematic polymer monolayers with the Onsager interaction potential. In the case of polymers with the Maier-Saupe potential which is an approximation to the Onsager potential, there is only one nematic branch. In contrast, polymers with the Onsager interaction potential have infinitely many nematic branches. For small $b$ (the normalized polymer concentration), the isotropic state is the only equilibrium. As $b$ is increased, these nematic branches appear one by one, starting with the first nematic branch at $b=(3 / 2) \pi$, then the second nematic branch at $b=(15 / 2) \pi$, then the third nematic branch at $b=(35 / 2) \pi$. The $j$-th nematic branch appears at $b=\left((2 j)^{2}-1\right) / 2 \pi$. For nematic states on the first branch, polymer rods are aligned along one direction, similar to nematic states in the case of the Maier-Saupe potential. Indeed, the phase diagram we obtained shows that the first nematic branch is close to the only nematic branch for the Maier-Saupe potential. For nematic states on the second branch, the behavior of the polymer orientation distribution is drastically different. Instead of being aligned along one direction, polymer rods are aligned with two perpendicular directions and the probability density has four fold symmetry. For nematic states on the $j$-th branch, polymer rods are aligned with $j$ directions uniformly distributed on the unit circle, and the probability density has $2 j$ fold symmetry. We use the leading non-zero coefficient in the Fourier expansion of the Onsager potential as an unnormalized order parameter. For the first nematic branch, the order parameter measures the alignment along the director (the direction along which the alignment is maximized), similar to the case of the Maier-Saupe potential. For the subsequent nematic branches, however, the order parameter should be viewed as a measure on the deviation of the probability density from the isotropic distribution. We have further designed a spectrum method for calculating the phase diagram of nematic polymers with the Onsager potential. The spectrum method is capable of producing numerical solutions that are accurate up to the computer precision, which we use as the exact solution. All asymptotic results obtained in this paper are confirmed by the numerical simulations. Although mathematically the asymptotic analysis applies only to nematic states with the Onsager potential of small amplitude, the comparison of the asymptotic results and numerical results demonstrates that the asymptotic expressions derived are still valid for a very large range of $b$, even when the amplitude of the Onsager potential is very large. We have also performed free energy calculations to study the stability of the isotropic branch and the nematic branches. Before the appearance of the first nematic branch at $b=(3 / 2) \pi$, the isotropic state is the only equilibrium state and is stable. For $b>(3 / 2) \pi$, the isotropic state is found to be unstable. The first nematic branch emerging at $b=(3 / 2) \pi$ has a free energy lower than that of the isotropic state, and thus is stable. All subsequent nematic branches are found to be unstable when perturbed by $\cos 2 \theta$, the leading Fourier mode in the first nematic branch. The fundamental difference between the Onsager potential and the Maier-Saupe potential is that for $j>1$ a perturbation of the Fourier mode $\cos 2 j \theta$ in the polymer orientation distribution will lead to a corresponding Fourier mode in the Onsager potential while it has no effect at all on the Maier-Saupe potential. In other words, for $j>1$ the Onsager potential responds to the Fourier mode $\cos 2 j \theta$ in the polymer orientation distribution while the Maier-Saupe potential does not. The $j$-th nematic branch can be viewed as nematic state excited by a perturbation of $\cos 2 j \theta$ from the isotropic state. Although 
all nematic branches with $j>1$ are unstable when perturbed by $\cos 2 \theta$ and consequently is physically not observable, the response of the Onsager interaction potential to the Fourier mode $\cos 4 \theta$ in the polymer orientation distribution is still significant. If an external field of the form of $\cos 4 \theta$ is applied, it will induce an amplitude of $\cos 4 \theta$ in the orientation distribution and the amplitude will be enhanced by the Onsager interaction potential.

Acknowledgments: This work was partially supported by the Air Force Office of Scientific Research under grant F1ATA06313G003 and by the National Science Foundation.

\section{References}

1. D. R. J. Chillingworth, E. Vicente Alonso, and A. A. Wheeler, J. Phys. 34, 1393 (2001).

2. F. Cocchini, C. Aratari, and G. Marruucci, Macromolecules 23, 4446 (1990).

3. P. Constantin, I. Kevrekidis, and E. S. Titi, Arch. Rat. Mech. Anal. 174, 365 (2004).

4. P. Constantin, I. Kevrekidis, and E. S. Titi, Discrete and Continuous Dynamical Systems 11, 101 (2004).

5. P. Constantin and J. Vukadinovic, Nonlinearity 18, 441 (2005).

6. M. Doi and S. F. Edwards, The Theory of Polymer Dynamics, Oxford University Press (1986).

7. V. Faraoni, M. Grosso, S. Crescitelli, and P. L. Maffetone, J. Rheol. 43, 829 (1999).

8. I. Fatkullin and V. Slastikov, Nonlinearity 18, 2565 (2005).

9. M. G. Forest, R. Zhou, and Q. Wang, Phys. Rev. E 66, 031712 (2002).

10. M. G. Forest, Q. Wang, and R. Zhou, Rheol. Acta 44, 80 (2004).

11. M. G. Forest, R. Zhou, and Q. Wang, Rheol. Acta 43, 17 (2004).

12. M. G. Forest, R. Zhou, and Q. Wang, Phys. Rev. Lett. 93, 088301 (2004).

13. M. G. Forest, R. Zhou, and Q. Wang, SIAM MMS 3, 853 (2005).

14. M. G. Forest, R. Zhou, and Q. Wang, J. Non-Newtonian Fluid Mech. 116, 183 (2004).

15. M. G. Forest and Q. Wang, Rheol. Acta 42, 20 (2003).

16. M. G. Forest, Q. Wang, R. Zhou, and E. Choate, J. Non-Newtonian Fluid Mech. 118, 17 (2004)
17. M. G. Forest, Q. Wang, and R. Zhou, J. Rheol. 51, 1 (2007).

18. D. Grecov and A. D. Rey, Rheologica Acta 42, 590 (2003).

19. A. Gopinath, L. Mahadevan, and R. C. Armstrong, Physics of Fluids 18, 028102 (2006).

20. S. Z. Hess, Z. Naturforsch. A 31A, 1034 (1976).

21. N. Kuzuu and M. Doi, Phys. Soc. Jpn. 52, 3486 (1983).

22. N. Kuzuu and M. Doi, Phys. Soc. Jpn. 53, 1031 (1984).

23. J. H. Lee, M. G. Forest, and R. Zhou, Discrete and Continuous Dynamical Systems-Series B 6, 339 (2006).

24. H. Liu, H. Zhang, and P. Zhang, Comm. Math. Sci. 3, 201 (2005).

25. C. Luo, H. Zhang, and P. Zhang, Nonlinearity 18, 379 (2005).

26. P. L. Maffettone and G. Marrucci, J. Non-Newtonian Fluid Mech. 38, 273 (1991)

27. P. L. Maffettone and S. Crescitelli, J. Rheol. 38, 1559 (1994).

28. P. L. Maffettone and S. Crescitelli, J. Non-Newtonian Fluid Mech. 59, 73 (1995).

29. P. L. Maffettone, M. Grosso, M. C. Friedenberg, and G. G. Fuller, Macromolecules 29, 8473 (1996).

30. G. Marrucci and P. L. Maffettone, Macromolecules 22, 4446 (1989).

31. G. Marrucci and P. L. Maffettone, J. Rheol. 34, 1217 (1990).

32. T. Maruyama, G. G. Fuller, M. Grosso, and P. L. Maffetone, J. NonNewtonian Fluid Mech. 76, 233 (1998).

33. L. Onsager, Ann. N. Y. Acad. Sci. 51, 627 (1949).

34. A. D. Rey and M. M. Denn, Annual Review of Fluid Mechanics 34, 233 (2002).

35. G. Rienäcker and S. Hess, Physica A 267, 294 (1999).

36. H. See, M. Doi, and R. G. Larson, J. Chem. Phys. 92, 792 (1990).

37. A. N. Semenov, Sov. Phys. JETP 66, 321 (1983).

38. G. Sgalari, G. L. Leal, and J. J. Feng, J. Non-Newtonian Fluid Mech. 102, 361 (2002).

39. E. Vicente Alonso, A. A. Wheeler, and T. J. Sluckin, Proc. R. Soc. Lond. A 459, 195 (2003).

40. H. Wang and H. Zhou, Phys. Lett. A 372, 3423 (2008).

41. Q. Wang, S. Sircar, and H. Zhou, Comm. Math. Sci. 3, 605 (2005).

42. K. S. Yim, G. G. Fuller, A. Datko, and C. Eisenbach, Macromolecules 34, 6972 (2001).

43. H. Yu and P. Zhang, J. Non-Newtonian Fluid Mech. 141, 116 (2007).

44. A. Zarnescu, Nonlinearity 19, 1619 (2006).

45. H. Zhou, H. Wang, M. G. Forest, and Q. Wang, Nonlinearity 18, 2815 (2005).

46. H. Zhou, H. Wang, Q. Wang, and M. G. Forest, Nonlinearity 20, 277 (2007).

47. H. Zhou and H. Wang, Comm. Math. Sci. 5, 113 (2007).

48. H. Zhou, H. Wang, and Q. Wang, Discrete and Continuous Dynamical Systems-Series B 7, 907 (2007).

49. H. Zhou and H. Wang, Physics of Fluids 19, 103107 (2007).

Received: 10 June 2008. Accepted: 30 December 2008. 